\title{
Moments of the Position of the Maximum for GUE Characteristic Polynomials and for Log-Correlated Gaussian Processes
}

\author{
Yan V. Fyodorov ${ }^{1}$. Pierre Le Doussal ${ }^{2}$
}

Received: 29 December 2015 / Accepted: 5 May 2016 / Published online: 19 May 2016

(C) The Author(s) 2016. This article is published with open access at Springerlink.com

\begin{abstract}
We study three instances of log-correlated processes on the interval: the logarithm of the Gaussian unitary ensemble (GUE) characteristic polynomial, the Gaussian log-correlated potential in presence of edge charges, and the Fractional Brownian motion with Hurst index $H \rightarrow 0$ (fBM0). In previous collaborations we obtained the probability distribution function (PDF) of the value of the global minimum (equivalently maximum) for the first two processes, using the freezing-duality conjecture (FDC). Here we study the PDF of the position of the maximum $x_{m}$ through its moments. Using replica, this requires calculating moments of the density of eigenvalues in the $\beta$-Jacobi ensemble. Using Jack polynomials we obtain an exact and explicit expression for both positive and negative integer moments for arbitrary $\beta>0$ and positive integer $n$ in terms of sums over partitions. For positive moments, this expression agrees with a very recent independent derivation by Mezzadri and Reynolds. We check our results against a contour integral formula derived recently by Borodin and Gorin (presented in the Appendix 1 from these authors). The duality necessary for the FDC to work is proved, and on our expressions, found to correspond to exchange of partitions with their dual. Performing the limit $n \rightarrow 0$ and to negative Dyson index $\beta \rightarrow-2$, we obtain the moments of $x_{m}$ and give explicit expressions for the lowest ones. Numerical checks for the GUE polynomials, performed independently by N. Simm, indicate encouraging agreement. Some results are also obtained for moments in Laguerre, Hermite-Gaussian, as well as circular and related ensembles. The correlations of the position and the value of the field at the minimum are also analyzed.
\end{abstract}

With Appendix 1 written by Alexei Borodin and Vadim Gorin.

$\bowtie \quad$ Yan V. Fyodorov

Y.Fyodorov@qmul.ac.uk

Pierre Le Doussal

pledoussal@yahoo.fr

1 School of Mathematical Sciences, Queen Mary University of London, London E1 4NS, UK

2 CNRS-Laboratoire de Physique Théorique de l'Ecole Normale Supérieure, 24 rue Lhomond, 75231 Paris Cedex, France 
Keywords Replica method · Freezing - Logarithmically correlated field · Statistics of minima $\cdot$ Random matrices $\cdot$ Jack polynomials

\section{Introduction}

Logarithmically correlated Gaussian (LCG) random processes and fields attract growing attention in mathematical physics and probability and play an important role in problems of statistical mechanics, quantum gravity, turbulence, financial mathematics and random matrix theory, see e.g. recent papers [1-5] for introduction and some background references and [6] for earlier review including condensed matter applications. A general lattice version of logarithmically correlated Gaussian field is a collection of Gaussian variables $V_{N, x}: x \in D_{N}$ attached to the sites of $d$-dimensional box $D_{N}$ of side length $N$ (assuming lattice spacing one) and characterized by the mean zero and the covariance structure

$$
\begin{aligned}
& \mathbb{E}\left\{V_{N, x}^{2}\right\}=2 g^{2} \log N+f(x), \\
& \mathbb{E}\left\{V_{N, x}, V_{N, y}\right\}=2 g^{2} \log _{+} \frac{N}{|x-y|}+\psi(x, y), \quad \text { for } x \neq y \in D_{N}
\end{aligned}
$$

where $\ln _{+}(w)=\max (\ln w, 0), g>0$ and both $f(x)$ and $\psi(x, y)$ are bounded function far enough from the boundary of $D_{N}$. One also can define the continuous versions $V(x)$ of LCG fields on various domains $D \in \mathbb{R}^{d}$ which is then necessarily a random generalized function ("random distribution"), the most famous example being the Gaussian Free field in $d=2$, see [7] for a rigorous definition. For $d=1$ the one-dimensional versions of LGC processes are known under the name of $1 / f$ noises, see e.g. [8,9]. They appear frequently in physics and engineering sciences, and also are rich and important mathematical objects of interest on their own. Such processes emerge, for example, in constructions of conformally invariant planar random curves [10] and are relevant in random matrix theory and studies of the Riemann zeta-function on the critical line [2].

In particular, the problem of characterizing the distribution of the global maximum $M_{N}=$ $\max _{x \in D_{N}} V_{N, x}$ of LCG fields and processes (or their continuum analogues) recently attracted a lot of interest, in physics, see [6,11-15] and mathematics, see [2,16-26]. The distribution is proved to be given by the Gumbel distribution with random shift [20] and has a universal tail predicted by renormalization group arguments in [6]. The detail of the full distribution are not universal and depend on some details of the behaviour of the covariance (2) for global $|x-y| \sim N$ scale as well as on the subleading term $f(x)$ in the variance (1). The explicit forms for the maximum distribution were conjectured in a few specific models of $1 / f$ noises $[2,11,12,21]$.

The goal of this paper is to provide some information about the distribution of the position of the global minimum

$$
x_{m}=\left\{x \in D: V(x)=\min _{y \in D} V(y)\right\}:=\operatorname{Arg} \min _{x \in D} V(x)
$$

for some examples of 1-dimensional processes with logarithmic correlations, though depending on applications, one can be interested instead in a maximum. Statistical properties of the value and position for maxima and minima are obviously trivially related in cases when $V(x)=-V(x)$ in law.

Our first example is the modulus of the characteristic polynomial of a random GUE matrix over the interval $[-1,1]$ of the spectral parameter. As is well-known, in the limit of large 
sizes of the matrix the logarithm of that modulus is very intimately related to $1 / f$ noises $[1,2,21,27,28]$. In that example the interesting quantity is obviously the statistics of the maximum, with minimum value being trivially zero at every characteristic root of the matrix. The second example is a general two-parameter variant of a log-correlated process on the interval with, in the language of Coulomb gases, endpoint charges, introduced and studied in [12]. That case may include a non-random (logarithmic) background potential $V_{0}(x)$, so that for the sum $V_{0}(x)+V(x)$ we have

$$
\min \left[V_{0}(x)+V(x)\right]=-\max \left[-V_{0}(x)+V(x)\right], \quad \text { in law }
$$

Our last example is a regularized version of the fractional Brownian motion with zero Hurst index, which is a bona fide (nonstationary) $1 d$ LCG process [1]. Here statistics of maxima and minima are trivially related by symmetry in law.

\section{Models, Method and Main Results}

We now define the three models to be considered. Although we did not yet succeed in obtaining the full probability distribution function (PDF), $\mathcal{P}\left(x_{m}\right)$, of the position of the global minimum $x_{m}$, we derive formulae for all positive integer moments of $x_{m}$ in terms of sums over partitions. In this section we present explicit values for some low moments of $x_{m}$, more results can be found in the remainder of the paper.

As is clear from [12] there is an intimate relation between statistics of extrema in logcorrelated fields on an interval and the $\beta$-Jacobi ensemble of random matrices $[29,30]$. In the course of our calculation we present methods to calculate the moments of the eigenvalue density of the Jacobi ensemble. In this section we provide a very explicit formula for these moments derived in remainder of the paper. In particular our result is in agreement with recent results by Mezzadri and Reynolds [31]. We check our results against a contour integral representation derived recently by Borodin and Gorin (presented in the Appendix 1 from these authors) which, remarkably, also allows to calculate negative moments.

Finally we sketch the replica method and the application of the freezing-duality conjecture to extract the moments of $x_{m}$ from the moments of the Jacobi ensemble.

\subsection{Results for Log-Correlated Processes}

\subsubsection{GUE Characteristic Polynomials (GUE-CP)}

Our first prediction is for the lowest moments of the position of the global maximum for the the modulus of the characteristic polynomial $p_{N}(x)=\operatorname{det}(x I-H)$ of the Hermitian $N \times N$ matrix $H$ sampled with the probability weight

$$
P(H) \propto \exp \left(-2 N \operatorname{Tr}\left(H^{2}\right)\right)
$$

known as the Gaussian Unitary Ensemble (or GUE) [32-34]. Here the variance is chosen to ensure that asymptotically for $N \rightarrow \infty$, the limiting mean density of the GUE eigenvalues is given by the Wigner semicircle law $\rho(x)=(2 / \pi) \sqrt{1-x^{2}}$ supported in the interval $x \in[-1,1]$. Hence the object we want to study is $\log \left|p_{N}(x)\right|=\sum_{i} \ln \left|x-\lambda_{i}\right|$ where the $\lambda_{i}$ are the eigenvalues of the GUE matrix $H$. To study its fluctuations it turns out to be more convenient to subtract its mean. This leads to the following 
Prediction 1 Define $\phi_{N}(x)=2 \log \left|p_{N}(x)\right|-2 \mathbb{E}\left(\log \left|p_{N}(x)\right|\right)$ and consider the random variable

$$
x_{m}^{(N)}:=\operatorname{Arg} \max _{x \in[-1,1]} \phi_{N}(x)
$$

Then the lowest even integer moments of this random variable have the values

$$
\lim _{N \rightarrow \infty} \mathbb{E}\left\{\left[x_{m}^{(N)}\right]^{2}\right\}=\frac{13}{49}, \quad \lim _{N \rightarrow \infty} \mathbb{E}\left\{\left[x_{m}^{(N)}\right]^{4}\right\}=\frac{20}{147}
$$

whereas the odd integer moments vanish by symmetry.

In particular, the kurtosis of the distribution of $x_{m}^{(N)}$ in the large- $N$ limit is given by

$$
\lim _{N \rightarrow \infty}\left(\frac{\mathbb{E}\left\{\left[x_{m}^{(N)}\right]^{4}\right\}}{\mathbb{E}\left\{\left[x_{m}^{(N)}\right]^{2}\right\}^{2}}-3\right)=-\frac{541}{507} \approx-1.067 \ldots
$$

To make a contact between $\left|p_{N}(x)\right|$ and the LCG processes we refer to the paper [1]. That work revealed that the natural large- $N$ limit of $\phi_{N}(x)$ is given by the random ChebyshevFourier series

$$
F(x)=-2 \sum_{n=1}^{\infty} \frac{1}{\sqrt{n}} a_{n} T_{n}(x), \quad x \in(-1,1),
$$

with $T_{n}(x)=\cos (n \arccos (x))$ being Chebyshev polynomials and real $a_{n}$ being independent standard Gaussian variables. A quick computation shows that the covariance structure associated with the generalized process $F(x)$ is given by an integral operator with kernel

$$
\mathbb{E}\{F(x) F(y)\}=4 \sum_{n=1}^{\infty} \frac{1}{n} T_{n}(x) T_{n}(y)=-2 \log (2|x-y|),
$$

as long as $x \neq y$. Such a limiting process $F(x)$ is an example of an aperiodic $1 / f$-noise.

Note however that the series (8) is formal and diverges with probability one. In fact it should be understood as a random generalized function (distribution). Though there is no sense in discussing the maxima and its position for generalized functions, the problem is well-defined for the logmod of the characteristic polynomial $\log \left|p_{N}(x)\right|$ for any finite $N$. One therefore needs to find a tool to utilize its asymptotically Gaussian nature evident in (8). It turns out that the latter is encapsulated in the following asymptotic formula due to Krasovsky $[35]^{1}$ which will be central for our considerations:

$$
\begin{aligned}
\mathbb{E}\left(\prod_{j=1}^{k}\left|p_{N}\left(x_{j}\right)\right|^{2 \delta_{j}}\right)= & \prod_{j=1}^{k} C\left(\delta_{j}\right)\left(1-x_{j}^{2}\right)^{\delta_{j}^{2} / 2}(N / 2)^{\delta_{j}^{2}} e^{\left(2 x_{j}^{2}-1-2 \log (2)\right) \delta_{j} N} \\
& \times \exp \left[-\sum_{1 \leq i<j \leq k} 2 \delta_{i} \delta_{j} \log \left|2\left(x_{i}-x_{j}\right)\right|\right]\left[1+O\left(\frac{\log N}{N}\right)\right]
\end{aligned}
$$

where $C(\delta):=2^{2 \delta^{2}} \frac{G(\delta+1)^{2}}{G(2 \delta+1)}$, with $G(z)$ being the Barnes G-function. In particular, differentiating with respect to $\delta$, we deduce that

$$
\mathbb{E}\left(2 \log \left|p_{N}(x)\right|\right)=N\left(2 x^{2}-1-2 \log (2)\right)+C^{\prime}(0)+O(\log (N) / N) .
$$

\footnotetext{
1 See also earlier works [36,37] where such formula was anticipated and proved for positive integer $\delta_{j}$.
} 
The formula (10) suggests that, apart from the factors $C\left(\delta_{j}\right)$ which as we shall see play no role in our calculations, the faithful description of $2 \log \left|p_{N}\left(x_{j}\right)\right|$ is that of the regularized GLC process with covariance (9), the position-dependent variance $2\left(\ln N+\ln \sqrt{1-x^{2}}-\ln 2\right)$ and the position-dependent mean $N\left(2 x^{2}-1-2 \log (2)\right)$. We find it convenient to subtract the mean value and concentrate on the centered GLC $\phi_{N}(x)$ in Prediction 1. As to the positiondependent logarithmic variance (stemming from the factors $\left(1-x_{j}^{2}\right)^{\delta_{j}^{2} / 2}$ in (10)) we shall see that it does play a very essential role in statistics of the position of global maximum for $\left|p_{N}(x)\right|$ via giving rise to nontrivial "edge charges" in the corresponding Jacobi ensemble. This observation corroborates with the earlier mentioned fact that the subleading position-dependent term $f(x)$ in the variance of the LCG, see (1), may modify the extreme value statistics.

\subsubsection{Log-Correlated Gaussian Random Potential (LCGP) with a background potential:}

An interesting question is to study the position of the minimum for the sum of a LCG random potential and of a determistic background potential, i.e:

$$
x_{m}=\operatorname{Argmin}_{x \in D}\left(V(x)+V_{0}(x)\right)
$$

Here we obtain results when $D$ is an interval, say $x \in[0,1]$. The LCG random potential has correlations:

$$
\mathbb{E}\left\{V(x) V\left(x^{\prime}\right)\right\}=C_{\epsilon}\left(x-x^{\prime}\right), \quad \lim _{\epsilon \rightarrow 0} C_{\epsilon}(x)=-2 \ln |x| \quad|x|>0
$$

and $C_{\epsilon}(0)=2 \ln (1 / \epsilon)$ where $\epsilon$ is a small scale regularization. The background potential is of the special logarithmic form:

$$
V_{0}(x)=-\bar{a} \ln x-\bar{b} \ln (1-x)
$$

which we will often refer to, following the Coulomb gas language, as "edge charges" at the boundary. We mainly focus on the case of repelling charges, $\bar{a}, \bar{b}>0$, although both the model, and some of our results, extend to some range of attractive charges. Some properties of this model, such as the PDF of the value of the total potential at the minimum, were studied in [12]. Here we obtain, for the two lowest moments of $x_{m}$

\section{Prediction 2}

$$
\begin{gathered}
\mathbb{E}\left\{x_{m}\right\}-\frac{1}{2}=\frac{\bar{a}-\bar{b}}{2(\bar{a}+\bar{b}+4)} \\
\mathbb{E}\left\{x_{m}^{2}\right\}-\left(\mathbb{E}\left\{x_{m}\right\}\right)^{2}=\frac{(\bar{a}+2)(\bar{b}+2)(2 \bar{a}+2 \bar{b}+9)}{(\bar{a}+\bar{b}+4)^{2}(\bar{a}+\bar{b}+5)^{2}}
\end{gathered}
$$

Note that for the background potential $V_{0}(x)$ alone, i.e. in the absence of disorder, and for $\bar{a}, \bar{b}>0$, the minimum for the background potential $V_{0}(x)$ alone is at $x_{m}^{0}=\frac{\bar{a}}{\bar{a}+\bar{b}}$, that is $x_{m}^{0}-\frac{1}{2}=\frac{\bar{a}-\bar{b}}{2(\bar{a}+\bar{b})}$. Hence the disorder brings the minumum closer in average to the midpoint $x=\frac{1}{2}$. 


\subsubsection{Fractional Brownian Motion with Hurst Index $H=0(f B m 0)$}

The fractional Brownian motion introduced by Kolmogorov in 1940 and rediscovered in the seminal work by Mandelbrot \& van Ness [38] is defined as the Gaussian process with zero mean and with the covariance structure:

$$
\mathbb{E}\left\{B_{H}\left(x_{1}\right) B_{H}\left(x_{2}\right)\right\}=\frac{\sigma_{H}^{2}}{2}\left(\left|x_{1}\right|^{2 H}+\left|x_{2}\right|^{2 H}-\left|x_{1}-x_{2}\right|^{2 H}\right),
$$

where $0<H<1$ and $\sigma_{H}^{2}=\operatorname{Var}\left\{B_{H}(1)\right\}$. The utility of these long-ranged correlated processes is related to the properties of being self-similar and having stationary increments, which characterize the corresponding family of Gaussian process uniquely. In particular, for $H=1 / 2$ the $\mathrm{fBm} B_{1 / 2}(t) \equiv B(t)$ is the usual Brownian motion (Wiener process). Note however that naively putting $H=0$ in (17) does not yield a well-defined process. Nevertheless we will see below that the limit $H \rightarrow 0$ for fractional Brownian motion can be properly defined after appropriate regularization and yields a Gaussian process with logarithmic correlations.

Consider a family of Gaussian processes depending on two parameters: $0 \leq H<1$ and a regularization $\eta>0$ and given explicitly by the integral representation [1]

$$
B_{H}^{(\eta)}(x)=\frac{1}{\sqrt{2}} \int_{0}^{\infty} \frac{e^{-\eta s}}{s^{1 / 2+H}}\left\{\left[e^{-i x s}-1\right] B_{c}(d s) / 2+\left[e^{i x s}-1\right] \overline{B_{c}(d s)} / 2\right\} .
$$

Here $B_{c}(s)=B_{R}(s)+i B_{I}(s)$, with $B_{R}(s)$ and $B_{I}(s)$ being two independent copies of the Wiener process $B(t)$ (the standard Brownian motion) so that $B(d t)$ is the corresponding white noise measure, $\mathbb{E}\{B(d t)\}=0$ and $\mathbb{E}\left\{B(d t) B\left(d t^{\prime}\right)\right\}=\delta\left(t-t^{\prime}\right) d t d t^{\prime}$.

The regularized process $\left\{B_{H}^{(\eta)}(x): x \in \mathbb{R}\right\}$ is Gaussian, has zero mean and is characterized by the covariance structure

$$
\mathbb{E}\left\{B_{H}^{(\eta)}\left(x_{1}\right) B_{H}^{(\eta)}\left(x_{2}\right)\right\}=\phi_{H}^{(\eta)}\left(x_{1}\right)+\phi_{H}^{(\eta)}\left(x_{2}\right)-\phi_{H}^{(\eta)}\left(x_{1}-x_{2}\right)
$$

where

$$
\begin{aligned}
\phi_{H}^{(\eta)}(x) & =\frac{1}{2} \int_{0}^{\infty} \frac{e^{-2 \eta s}}{s^{1+2 H}}(1-\cos (x s)) d s \\
& =\frac{1}{4 H} \Gamma(1-2 H)\left[\left(4 \eta^{2}+x^{2}\right)^{H} \cos \left(2 H \arctan \frac{x}{2 \eta}\right)-(2 \eta)^{2 H}\right] .
\end{aligned}
$$

It is easy to verify that for any $0<H<1$ one has $\lim _{\eta \rightarrow 0} B_{H}^{(\eta)}(x)=B_{H}(x)$ which is precisely the fBm defined in (17).

As has been already mentioned the limit $\eta \rightarrow 0$ for $H=0$ does not yield any well-defined process. At the same time taking the limit $H \rightarrow 0$ at fixed $\eta$ gives

$$
\lim _{H \rightarrow 0} \phi_{H}^{(\eta)}(x)=\frac{1}{4} \log \frac{x^{2}+4 \eta^{2}}{4 \eta^{2}},
$$

ensuring that for any $\eta>0$ the limit of $B_{H}^{(\eta)}(x)$ as $H \rightarrow 0$ yields a well-defined Gaussian process $\left\{B_{0}^{(\eta)}(x): x \in \mathbb{R}\right\}$ with stationary increments and with the increment structure function depending logarithmically on the time separation:

$$
\mathbb{E}\left\{\left[B_{0}^{(\eta)}\left(x_{1}\right)-B_{0}^{(\eta)}\left(x_{2}\right)\right]^{2}\right\}=\frac{1}{2} \log \frac{\left|x_{1}-x_{2}\right|^{2}+4 \eta^{2}}{4 \eta^{2}} .
$$


We consider $B_{0}^{(\eta)}(x)$ as the most natural extension of the standard fBm to the case of zero Hurst index $H=0$. We will frequently refer to this process as $\mathrm{fBm} 0$. The process is regularized at scales $\left|x_{1}-x_{2}\right|<2 \eta$.

It is also worth pointing out that there exists an intimate relation between $B_{0}^{(\eta)}(x)$ and the behaviour of the (increments of) GUE characteristic polynomials, though at a different, socalled "mesoscopic" spectral scales [1], negligible in comparision with the interval $[-1,1]$. The mesoscopic intervals are defined as those typically containing in the limit $N \rightarrow \infty$ a number of eigenvalues growing with $N$, but representing still a vanishingly small fraction of the total number $N$ of all eigenvalues. In other words, fBM0 describes behaviour of the (logarithm of) the ratio of the moduli of characteristic polynomial at mesoscopic difference in spectral parameter. More precisely, $B_{0}^{(\eta)}(x)$ is , in a suitable sense, given by $N \rightarrow \infty$ limit of the following object:

$$
W_{N}(x)=\frac{1}{2 \pi}\left(-\log \operatorname{det}\left[\left(\frac{x}{d_{N}} I-H\right)^{2}+\frac{\eta^{2}}{4 d_{N}^{2}}\right]+\log \operatorname{det}\left[H^{2}+\frac{\eta^{2}}{4 d_{N}^{2}}\right]\right)
$$

where $H$ is an $N \times N$ random GUE matrix, parameter $\eta>0$ is a regularisation ensuring that the logarithms are well defined for real $x$ and $d_{N}$ specifies the asymptotic scale of the spectral axis of $H$ as $N \rightarrow \infty$ and is chosen to be mesoscopic $1 \ll d_{N} \ll N$ (say, $d_{N}=N^{\gamma}$ with $0<\gamma<1)$.

Applying our methods of dealing with LCG to fbM0 yields the following predictions for a few lowest moments of the position of the global minimum for the process $B_{0}^{(\eta)}(x)$ in the interval $0, L$ :

Prediction 3 Define $V(x)=2 B_{0}^{(\eta)}(x)$ and for $\eta>0$ and $L>0$ consider the random variable

$$
y_{m}(\eta, L):=\frac{1}{L} \operatorname{Arg} \min _{x \in[0, L]} V(x)
$$

Then the lowest even integer moments of this random variable have the values

$$
\lim _{\eta \rightarrow 0} \mathbb{E}\left\{\left[y_{m}(\eta, L)\right]^{2}\right\}=\frac{17}{50}, \quad \lim _{\eta \rightarrow 0} \mathbb{E}\left\{\left[y_{m}(\eta, L)\right]^{4}\right\}=\frac{311}{1470}
$$

whereas the odd integer moments can be found from the identities

$$
\lim _{\eta \rightarrow 0} \mathbb{E}\left\{\left[y_{m}(\eta, L)-\frac{1}{2}\right]^{2 k+1}\right\}=0, \quad k=0,1,2, \ldots
$$

One should point out an interesting difference in application of our method to this case, concerning the value of the minimum $V_{m}=\min _{x \in[0,1]} V(x)$, which seems to be a direct consequence of non-stationarity of the $\mathrm{fBm} 0$. Since the process is constrained to the value $V(0)=2 B_{0}^{(\eta)}(0)=0$ at zero, $V_{m}$ is necessarily negative or zero, at variance with the other cases studied here. As discussed in Appendix 7, this implies that the method of analytical continuation in $n$ of Ref. [12], which works nicely for the other cases, fails to predict the PDF of $V_{m}$ for the fBm0, and requires modifications which are left for future studies. We do not believe that this problem bears consequence to the moments of $x_{m}$, which enjoy a nice and simple analytical continuation to $n=0$. As we checked numerically up to large $n$, these moments pass the standard tests (i.e. positivity of Hankel matrices) for existence of a positive associated PDF. Conditional moments however, i.e. conditioned to an atypically high value of $V_{m}$, would need a more careful study, beyond the scope of this paper. 


\subsection{Moments of the Eigenvalue Density of the Jacobi Ensemble}

As mentioned above the statistics of extrema in log-correlated fields on an interval relate to the $\beta$-Jacobi ensemble of random matrices. We denote $\mathbf{y}=\left(y_{1}, \ldots, y_{n}\right)$ the set of eigenvalues, with $y_{i} \in[0,1], i=1, \ldots, n$. The model can be defined $[29,30]$ by the joint distribution of eigenvalues $^{2}$

$$
\mathcal{P}_{J}(\mathbf{y}) d \mathbf{y}=\frac{1}{\mathcal{Z}_{n}} \prod_{i=1}^{n} d y_{i} y_{i}^{a}\left(1-y_{i}\right)^{b}|\Delta(\mathbf{y})|^{2 \kappa}, \quad \Delta(\mathbf{y})=\prod_{1 \leq i<j \leq n}\left(y_{i}-y_{j}\right)
$$

where the normalization constant, $\mathcal{Z}_{n}$, is the famous Selberg integral [39] for which an explicit formula exists for any positive integer $n$

$$
\begin{aligned}
\mathcal{Z}_{n} & =S l_{n}(\kappa, a, b):=\int_{[0,1]^{n}}|\Delta(\mathbf{y})|^{2 \kappa} \prod_{i=1}^{n} y_{i}^{a}\left(1-y_{i}\right)^{b} d y_{i} \\
& =\prod_{j=0}^{n-1} \frac{\Gamma(a+1+\kappa j) \Gamma(b+1+\kappa j) \Gamma(1+\kappa(j+1))}{\Gamma(a+b+2+\kappa(n+j-1)) \Gamma(1+\kappa)}
\end{aligned}
$$

In [12] we analytically continued this formula to complex $n$ which allowed to obtain the probability distribution of the height of the global minimum of the process (see also [4043]). In the present paper we advance this analysis much further in order to extract the statistics of the position of the global minimum. As we will show this requires to obtain some exact formula for the moments of the eigenvalue density for the $\beta$-Jacobi ensemble for arbitrary positive integer $n$. Furthermore these should be explicit enough to allow for a continuation to $n=0$.

Let us define the average value $<f(y)>_{J}$ of any function $f(y)$ over the Jacobi density by the relation

$$
<f(\mathbf{y})>_{J}:=\left[S l_{n}(\kappa, a, b)\right]^{-1} \int_{[0,1]^{n}} f(\mathbf{y})|\Delta(\mathbf{y})|^{2 \kappa} \prod_{i=1}^{n} y_{i}^{a}\left(1-y_{i}\right)^{b} d y_{i}
$$

In particular for any positive integer $n$ the mean density of eigenvalues is defined as:

$$
\rho_{J}(y)=<\frac{1}{n} \sum_{i=1}^{n} \delta\left(y_{i}-y\right)>_{J}=<\delta\left(y_{1}-y\right)>_{J}
$$

where $\delta$ is the Dirac distribution. The moments studied here are then defined as:

$$
M_{k}^{(J)}=\int_{0}^{1} d y y^{k} \rho_{J}(y)=<y_{1}^{k}>_{J}
$$

The problem of calculating these moments, for the $\beta$-Jacobi ensemble, has been already addressed in the theoretical physics and mathematical literature, motivated by various applications. In full generality it turns out to be a hard problem, and only limited results were available. One method is based on recursion on the order of the moment, as outlined in the

2 Note that we use $2 \kappa$ for the Dyson index instead of $\beta$ to avoid confusion with the inverse temperature, also denoted $\beta$, associated to the study of the log-correlated process. 
chapter 17 of Mehta's book [33] and used in [44,45] in the context of conductance distribution in chaotic transport through mesoscopic cavities. In that approach higher moments calculations become technically unsurmountable. Another approach using Schuhr functions was developed and gave very explicit results for these and other moments for $\beta=1,2$ [46] (see also related work in [47] and [48]).

More recently, an interesting contour integral representation for those moments was proved by Borodin and Gorin, but remained unpublished. It is described in the Appendix 1 to the present paper, provided by these authors. It allows a systematic calculation of the moments, including negative ones. However evaluating these integrals becomes again a challenge for higher moments.

In the present paper we give an explicit expression for all integer moments, positive and negative, of the eigenvalue density for the $\beta$-Jacobi ensemble, based on a different approach, in terms of sums over partitions.

Let us further denote $\lambda=\left(\lambda_{1} \geq \lambda_{2} \geq \cdots \geq \lambda_{\ell(\lambda)}\right)$ a partition of length $\ell(\lambda)$ of the integer $k \geq 0$, with $\lambda_{i}$ strictly positive integers such that $|\lambda|=\sum_{i=1}^{\ell(\lambda)} \lambda_{i}=k$. Then we obtain

$$
\left\langle\frac{1}{n} \sum_{j=1}^{n} y_{j}^{k}\right\rangle_{J}=\sum_{\lambda,|\lambda|=k} A_{\lambda} a_{\lambda}^{+},\left\langle\frac{1}{n} \sum_{j=1}^{n} y_{j}^{-k}\right\rangle_{J}=\sum_{\lambda,|\lambda|=k} A_{\lambda} a_{\lambda}^{-}
$$

where the sum is over all partitions of $k$, and

$$
\begin{aligned}
A_{\lambda}= & \frac{k\left(\lambda_{1}-1\right) !}{(\kappa(\ell(\lambda)-1)+1)_{\lambda_{1}}} \prod_{i=2}^{\ell(\lambda)} \frac{(\kappa(1-i))_{i}}{(\kappa(\ell(\lambda)-i)+1)_{\lambda_{i}}} \prod_{1 \leq i<j \leq \ell(\lambda)} \frac{\kappa(j-i)+\lambda_{i}-\lambda_{j}}{\kappa(j-i)} \\
& \times \frac{1}{n} \prod_{i=1}^{\ell(\lambda)} \frac{(\kappa(n-i+1))_{\lambda_{i}}}{(\kappa(\ell(\lambda)-i+1))_{\lambda_{i}}} \prod_{1 \leq i<j \leq \ell(\lambda)} \frac{(\kappa(j-i+1))_{\lambda_{i}-\lambda_{j}}}{(\kappa(j-i-1)+1) \lambda_{i}-\lambda_{j}}
\end{aligned}
$$

in terms of the Pochhammer symbol $(x)_{n}=x(x+1) \cdots(x+n-1)=\Gamma(x+n) / \Gamma(x)$, with for positive moments

$$
a_{\lambda}^{+}=\prod_{i=1}^{\ell(\lambda)} \frac{(a+1+\kappa(n-i))_{\lambda_{i}}}{(a+b+2+\kappa(2 n-i-1))_{\lambda_{i}}}
$$

while for negative moments

$$
a_{\lambda}^{-}=\prod_{i=1}^{\ell(\lambda)} \frac{(a+1+\kappa(i-1))_{-\lambda_{i}}}{(a+b+2+\kappa(n+i-2))_{-\lambda_{i}}}
$$

This result as it stands was derived for $n, k \in \mathbb{N}$ and $\kappa>0$, and in range of values of $a, b$ such that these moments exist. Later however we will study analytic continuations in these parameters.

For positive moments, this very explicit formula is equivalent to a result by Mezzadri and Reynolds, which appeared in [31] during the course of the present work. Our independent derivation is relatively straightforward and will be given below for both positive and negative moments.

One can check on the above expressions $(33,34)$, that in the limit $\kappa \rightarrow 0$ partitions contribute as $\sim \kappa^{\ell(\lambda)-1}$, hence only the single partition $\lambda=(k)$ with a single row contributes, leading to the trivial limit for the moments: 


$$
\left\langle\frac{1}{n} \sum_{j=1}^{n} y_{j}^{k}\right\rangle_{J, \kappa=0}=\frac{(a+1)_{k}}{(a+b+2)_{k}}
$$

here for $k$ of either sign, as expected, since in that limit the Jacobi measures decouples $\mathcal{P}_{J}(\mathbf{y})=\prod_{i=1}^{n} P^{0}\left(y_{i}\right)$ where $P^{0}(y)=\frac{\Gamma(a+b+2)}{\Gamma(a+1) \Gamma(b+1)} y^{a}(1-y)^{b}$. Other general properties of the moments, such as identity of moments of $y$ and of $1-y$ for $a=b$, are less straightforward to see on the above formula, and are explicitly checked below for low moments.

\subsection{Replica Method and Freezing Duality Conjecture for Log-Correlated Processes}

Our method of addressing statistics related to the global minimum of random functions (which can be trivially adapted for the global maximum with obvious modifications) is inspired by statistical mechanics of disordered systems. Namely, we look at any random function $V(x)$ defined in an interval $D \in \mathbb{R}$ of the real axis as a one-dimensional random potential, with $x$ playing the role of spatial coordinate. To that end we introduce for any $\beta>0$ and any positive $\beta$-independent weight function $\mu(x)>0$ the associated Boltzmann-Gibbs-like equilibrium measure by

$$
p_{\beta}(x)=\frac{1}{Z_{\beta}} \mu(x) e^{-\beta V(x)} \equiv \frac{1}{Z_{\beta}} \int_{D} \delta\left(x-x_{1}\right) e^{-\beta V\left(x_{1}\right)} \mu\left(x_{1}\right) d x_{1},
$$

where we have defined the associated normalization function (the "partition function")

$$
Z_{\beta}=\int_{D} e^{-\beta V(x)} \mu(x) d x
$$

with $\beta=1 / T$ playing the role of inverse temperature.

According to the basic principles of statistical mechanics in the limit of zero temperature $\beta \rightarrow \infty$ the Boltzmann-Gibbs measure must be dominated by the minimum of the random potential $V_{m}=\min _{x \in D} V(x)$ achieved at the point $x_{m} \in D$. The latter is randomly fluctuating from one realization of the potential to another. The probability density for the position of the minimum is defined as $\mathcal{P}(x)=\overline{\delta\left(x-x_{m}\right)}$ where from now on we use the bar to denote the expectation with respect to random process ("potential disorder") realizations:

$$
\overline{(\ldots)} \equiv \mathbb{E}\{(\ldots)\}
$$

This leads to the fundamental relation

$$
\mathcal{P}(x)=\lim _{\beta \rightarrow \infty} \overline{p_{\beta}(x)}
$$

Therefore, calculating $\mathcal{P}(x)$ amounts to (i) performing the disorder average of the BoltzmannGibbs measure (38) and (ii) evaluating its zero-temperature limit. The second step is highly non-trivial, due to a phase transition occuring at some finite value $\beta=\beta_{c}$. In our previous work on decaying Burgers turbulence [49], which turns out to be a limiting case of the present problem (see Sect. 4.3), we have already succeeded in implementing that program. Following the same strategy, the step (i) is done using the replica method, a powerful (albeit not yet mathematically rigorous) heuristic method of theoretical physics of disordered systems. It amounts to representing $Z_{\beta}^{-1}=\lim _{n \rightarrow 0} Z_{\beta}^{n-1}$ which after assuming integer $n>1$ results in the formal identity

$$
\overline{p_{\beta}(x)}=\lim _{n \rightarrow 0} p_{\beta, n}(x)
$$


where

$$
p_{\beta, n}(x)=\mu(x) \overline{e^{-\beta V(x)} Z_{\beta}^{n-1}}=\int_{x_{1} \in D} \cdots \int_{x_{n} \in D} \overline{e^{-\beta \sum_{=1}^{n} V\left(x_{i}\right)}} \delta\left(x-x_{1}\right) \prod_{i=1}^{n} \mu\left(x_{i}\right) d x_{i}
$$

Note that $p_{\beta, n}(x)$ is not a probability distribution for general $n$, but becomes one for $n=0$.

In the next section we will show how to calculate the moments $M_{k}(\beta)=\int_{D} p_{\beta, n}(x) x^{k} d x$ for positive integer $k$ and any integer value $n>0$ in the high temperature phase of the model $\beta<\beta_{c}$. Note that by a trivial rescaling of the potential one always can ensure $\beta_{c}=1$, setting $g=1$ in (1), and we assume such a rescaling henceforth. In the range $\beta<1$ the formulae we obtain for the moments turn out to be easy to continue to $n=0$. This yields the integer moments of the probability density $\overline{p_{\beta}(x)}$ in that phase. There still however remains the task of finding a way to continue those expressions to $\beta>1$ in order to compute the limit $\beta \rightarrow \infty$ and extract the information about the Argmin distribution $\mathcal{P}(x)$. To perform the continuation, we rely on the freezing transition scenario for logarithmically correlated random landscapes. The background idea goes back to [6] and was advanced further in [11] leading to explicit predictions. In [12] it was discovered that the duality property appears to play a crucial role, leading to the freezing-duality conjecture (FDC), which was further utilized in $[2,9,13,14,21,49]$. In brief, the FDC predicts a phase transition at the critical value $\beta=1$ and amounts to the following principle:

Thermodynamic quantities which for $\beta<1$ are duality-invariant functions of the inverse temperature $\beta$, that is remain invariant under the transformation $\beta \rightarrow \beta^{-1}$, "freeze" in the low temperature phase, that is retain for all $\beta>1$ the value they acquired at the point of self-duality $\beta=1$.

Here, on our explicit formula, we will indeed be able to verify that every integer moment $M_{k}(\beta)=\int_{D} p_{\beta, n=0}(x) x^{k} d x$ of the probability density $\overline{p_{\beta}(x)}$ is duality-invariant in the above sense, and hence can be continued to $\beta>1$ using the FDC, yielding the moments of the position of the global minimum. Another way of proof is based on the powerful contour integral representation for the moments of Jacobi ensemble of random matrices provided by Borodin and Gorin. We thus conjecture that not only all moments, but the whole disorder averaged Gibbs measure $\overline{p_{\beta}(x)}$ freezes at $\beta=1$, hence that the PDF of the position of the minimum is determined as

$$
\mathcal{P}(x)=\lim _{\beta \rightarrow 1} \overline{p_{\beta}(x)}
$$

similar to the conjecture in [49] in our study of the Burgers equation.

Although the FDC scenario is not yet proven mathematically in full generality and has a status of a conjecture supported by physical arguments and available numerics, recently a few nontrivial aspects of freezing were verified within rigorous probabilistic analysis, see e.g. $[20,22,23,50]$ for progress in that direction. ${ }^{3}$ However the role of duality has not yet been verified rigorously. Interesting connections to duality in Liouville and conformal field theory [51] remain to be clarified.

\footnotetext{
${ }^{3}$ In $[6,11,12]$ we predicted the freezing of the generating function $g_{\beta}(y):=\overline{\exp \left(-e^{\beta y} z_{\beta}\right)}$, where $z_{\beta}$ is a regularized version of the partition sum $Z_{\beta}$. It was proved in [23] (see corollary 2.3) in a more general and rigorous setting. It tells about the free energy $f_{\beta}=-\beta^{-1} \ln z_{\beta}$ and the value of the minimum $f_{\infty}$. Indeed, by construction $1-g_{\beta}(y)$ is the cumulative distribution function of the random variable defined as $y_{\beta}:=f_{\beta}-\beta^{-1} G$ where $G$ is a unit Gumbell random variable, independent from $f_{\beta}$. The PDF of $y_{\beta}$ for any $\beta<\beta_{c}$, of $y_{\beta_{c}}$ and of $f_{\infty}$ are thus identical. See the Appendix 8 for more details.
} 
In the rest of the paper we give a detailed derivation of the outlined steps of our procedure and an analysis of the results.

\section{Calculations Within the Replica Method}

Our goal in this section is to develop the method of evaluating the required disorder average and calculating the resulting integrals explicitly in some range of inverse temperatures $\beta$ for a few instances of the log-correlated random potentials $V(x)$.

\subsection{Connections to the $\beta$-Jacobi Ensemble}

\subsubsection{GUE Characteristic Polynomial}

In that case we will follow the related earlier study in [21] and use the family of weight factors $\mu(x)=\rho(x)^{q}$ on the interval $x \in[-1,1]$, with $\rho(x)=\frac{2}{\pi} \sqrt{1-x^{2}}$ and parameter $q>0$. Such a choice of the weight is justified a posteriori by the possibility to find within this family a duality-invariant expression for the moments in the high-temperature phase which is central for our method to work. Since here we are interested in the maximum of the characteristic polynomial we will define the potential $V(x)=-\phi_{N}(x)$ where $\phi_{N}(x)$, defined in Prediction 1, is not strictly a Gaussian field. Nevertheless due to the Krasovsky formula (10) we can write asymptotically in the limit of large $N \gg 1$

$$
\begin{aligned}
& \overline{e^{-\beta \sum_{a=1}^{n} V\left(x_{a}\right)}}=\overline{e^{\beta \sum_{a=1}^{n} \phi_{N}\left(x_{a}\right)}}=\overline{\prod_{a=1}^{n}\left|p_{N}\left(x_{a}\right)\right|^{2 \beta}} e^{-2 \beta \sum_{a=1}^{n} \overline{\ln \left|p_{N}\left(x_{a}\right)\right|}} \\
& \simeq A_{n} \prod_{a=1}^{n}\left(1-x_{a}^{2}\right)^{\beta^{2} / 2} \prod_{a<b}\left|x_{a}-x_{b}\right|^{-2 \beta^{2}}
\end{aligned}
$$

with $A_{n}=\left[C(\beta)(N / 2)^{\beta^{2}} e^{-\beta C^{\prime}(0)} 2^{-\beta^{2}(n-1)}\right]^{n}$.

\subsubsection{General Scaled Model in [0, 1].}

It is easy to see that by proper rescaling the evaluation of the function $p_{\beta, n}(x)$ from (42) amounts in the case of both characteristic GUE polynomials and the LCGP process to evaluating particular cases of the following multiple integral defined on the interval $[0,1]$ :

$$
p_{\beta, a, b, n}(y)=\int_{0}^{1} \cdots \int_{0}^{1} \prod_{i=1}^{n} d y_{i} y_{i}^{a}\left(1-y_{i}\right)^{b} \prod_{1 \leq i<j \leq j \leq n} \frac{1}{\left|y_{i}-y_{j}\right|^{2 \beta^{2}}} \delta\left(y-y_{1}\right)
$$

which is formally the (un-normalized) density of eigenvalues of the $\beta$-Jacobi ensemble (28), i.e. $p_{\beta, a, b, n}(y)=\mathcal{Z}_{n} \rho_{J}(y)$, however with a negative value of the parameter $\kappa=-\beta^{2}$. In particular the normalisation factor $\mathcal{Z}_{n}$ is the Selberg integral (29). Note that both integrals are well defined for $\beta^{2}<1$ and positive integer $n$. They can also be defined for larger $\beta$, upon introducing an implicit small scale cutoff which modifies the expressions for $\left|y_{i}-y_{j}\right|<\epsilon$. However we will use only the high temperature regime and will continue analytically our moment formula to $n=0$ and $\beta=1$.

These integrals are associated to the statistical mechanics of a random energy model generated by a LCG field in the interval $[0,1]$ in presence of boundary charges of strength $a$ and $b$. More precisely, they appear in the study of the continuum partition function introduced in [12] 


$$
Z_{\beta}=\epsilon^{\beta^{2}} \int_{0}^{1} d y y^{a}(1-y)^{b} e^{-\beta V(y)}
$$

where the correlator of $V(x)$ was defined in (13). Here $a$ and $b$ are the two parameters of the model and the factor $\epsilon^{\beta^{2}}$ ensures that the integer moments $\overline{Z_{\beta}^{n}}$ are $\epsilon$-independent in the high temperature regime. There, these moments $\overline{Z_{\beta}^{n}}=\mathcal{Z}_{n}$ are given by the Selberg integral (29). Several aspects of this model, such as freezing, duality and obtaining the PDF of the value of the minimum, were analyzed in [12]. Here we will focus instead on the calculation of the moments of the position $y$. In each realization of the random potential $V$ they are defined as

$$
<y^{k}>_{\beta, a, b}=\frac{\int_{0}^{1} d y y^{k} y^{a}(1-y)^{b} e^{-\beta V(y)}}{\int_{0}^{1} d y y^{a}(1-y)^{b} e^{-\beta V(y)}}
$$

and we will be interested in calculating their disorder averages $\overline{<y^{k}>_{\beta, a, b}}$.

\subsubsection{Fractional Brownian Motion}

For our $\mathrm{fBm} 0$ example we take the weight function $\mu(x)=1$ in $(38,39)$ on the interval $x \in[0, L]$, and use the rescaled fBm with $H=0$ as the random potential: $V(x)=2 g B_{0}^{(\eta)}(x)$. Exploiting the Gaussian nature of $\mathrm{fBm} 0$ we can easily perform the required average using (19) which yields

$$
\begin{aligned}
\overline{e^{-2 \beta g \sum_{i=1}^{n} B_{0}^{(\eta)}\left(x_{i}\right)}} & =e^{2(\beta g)^{2}\left\{\sum_{i=1}^{n} \overline{\left[B_{0}^{(\eta)}\left(x_{i}\right)\right]^{2}}+2 \sum_{i<j}^{n} \overline{B_{0}^{(\eta)}\left(x_{i}\right) B_{0}^{(\eta)}\left(x_{j}\right)}\right\}} \\
& =e^{n(2 \beta g)^{2} \sum_{i=1}^{n} \phi_{0}^{(\eta)}\left(x_{i}\right)-(2 \beta g)^{2} \sum_{i<j}^{n} \phi_{0}^{(\eta)}\left(x_{i}-x_{j}\right)}
\end{aligned}
$$

Further using the definitions $(22,23)$ we arrive at

$$
\begin{aligned}
& \overline{e^{-2 \beta g \sum_{i=1}^{n} B_{0}^{(\eta)}\left(x_{i}\right)}}=\prod_{i=1}^{n}\left[\frac{x_{i}^{2}+4 \eta^{2}}{4 \eta^{2}}\right]^{a / 2} \prod_{i<j}^{n}\left[\frac{\left(x_{i}-x_{j}\right)^{2}+4 \eta^{2}}{4 \eta^{2}}\right]^{-\gamma} \\
& a=2 n \gamma, \quad \gamma=g^{2} \beta^{2}
\end{aligned}
$$

Assuming that all $x_{i}>0$ we can write in the limit of vanishing regularization $\eta \rightarrow 0$ :

$$
\overline{e^{-2 \beta g \sum_{i=1}^{n} B_{0}^{(\eta)}\left(x_{i}\right)}} \approx(2 \eta)^{n(\gamma(n-1)-a)} \prod_{i=1}^{n} x_{i}^{a} \prod_{i<j}^{n}\left|x_{i}-x_{j}\right|^{-2 \gamma}
$$

For convenience we will use the particular value $g=1$ ensuring a posteriori the critical temperature value $\beta_{c}=1$. (46):

It is easy to see how our three examples can be studied within the framework of the model

- GUE characteristic polynomial. We define $x=1-2 y$, with $y \in[0,1]$ and

$$
\begin{aligned}
& p_{\beta, n}(x) d x=C_{n} p_{\beta, a, a, n}(y) d y, \quad a=\frac{q+\beta^{2}}{2} \\
& C_{n}=\left[\left(\frac{2}{\pi}\right)^{q} C(\beta) N^{\beta^{2}} e^{-\beta C^{\prime}(0)} 2^{1+q-2 \beta^{2}(n-1)}\right]^{n}
\end{aligned}
$$

- LCGP plus a background potential on interval [0, 1], defined in (13) and (14). One sees that this model is obtained by choosing $a=\beta \bar{a}$ and $b=\beta \bar{b}$. 
- fBm0: we define $x=L y$, with $y \in[0,1]$ and:

$$
p_{\beta, n}(x) d x=L^{n(n+1) \gamma+n} p_{\beta, a=2 n \beta^{2}, b=0, n}(y) d y
$$

Although the explicit form of the density $\rho_{J}(y)$ of the $\beta$-Jacobi ensemble is not known in a closed form for finite $n$, the formulae $(33,34)$ displayed in the introduction provide an explicit expression for its integer moments

$$
<y^{k}>_{\beta, a, b, n}:=\frac{1}{\mathcal{Z}_{n}} \int_{0}^{1} d y y^{k} p_{\beta, a, b, n}(y)
$$

for positive integers $k, n$ and $\beta^{2}<0$. The collection of indices $\beta, a, b, n$ is now replacing the index $J$, and some of them may be omitted below when no confusion is possible.

We will now derive the formulae $(33,34)$ using methods based on Jack polynomials. In a second stage we will continue them analytically to arbitrary $n$, including $n=0$, and to $0<\beta^{2} \leq 1$, to obtain moments for the random model of interest as:

$$
\overline{<y^{k}>_{\beta, a, b}}=\lim _{n \rightarrow 0}<y^{k}>_{\beta, a, b, n}
$$

\subsection{Derivation of the Moment Formula in Terms of Sums over Partitions}

To calculate the moments, we now consider one of the most distinguished bases of symmetric polynomials, the Jack polynomials, named after Henry Jack. They play a central role for our study because their average with respect to the $\beta$-Jacobi measure is explicitly known, due to Kadell [52] (see below). As discussed in the book [53] (see reprint of the original article) Jack introduced a special set of symmetric polynomials of $n$ variables $\mathbf{y}:=\left(y_{1}, \ldots, y_{n}\right)$ indexed by integer partitions $\lambda$ and dependent on a real parameter $\alpha$. He called them $Z(\lambda)$, which in modern notations are denoted as $J_{\lambda}^{(\alpha)}(\mathbf{y})$ following I. Macdonald who greatly developed the theory of such and related objects in the book [54]. Another important source of information is Stanley's paper [55]. In what follows we use notations and conventions from [54] and [55]. ${ }^{4}$

Let us recall the definition of a partition $\left.\lambda=\left(\lambda_{1} \geq \lambda_{2} \geq \cdots \geq \lambda_{\ell(\lambda)}\right)>0\right)$ of the integer $k$, of length $\ell(\lambda)$, with $\lambda_{i}$ strictly positive integers such that $|\lambda|:=\sum_{i=1}^{\ell(\lambda)} \lambda_{i}=k$. It can be written as

$$
\lambda=\left\{(i, j) \in \mathbb{Z}^{2} ; 1 \leq i \leq \ell(\lambda), 1 \leq j \leq \lambda_{i}\right\}
$$

from which one usually draw the Young diagram representing the partition $\lambda$, as a collection of unit area square boxes in the plane, centered at coordinates $i$ along the (descending, southbound) vertical, and $j$ along the (eastbound) horizontal. The dual (or conjugate) $\lambda^{\prime}$ of $\lambda$ is the partition whose Young diagram is the transpose of $\lambda$, i.e. reflected along the (descending) diagonal $i=j$. Hence $\lambda_{i}^{\prime}$ is the number of $j$ such that $\lambda_{j} \geq i$. If $s=(i, j)$ stands for a square in the Young diagram, one defines the "arm length" $a_{\lambda}(s)=\lambda_{i}-j$ which is equal to the number of squares to the east of square $s$ and the "leg length" $l_{\lambda}(s)=\lambda_{j}^{\prime}-i$ as the number of squares to the south of the square $s$. One then defines the product

$$
c(\lambda, \alpha, t):=\prod_{s \in \lambda}\left(\alpha a_{\lambda}(s)+l_{\lambda}(s)+t\right)=\prod_{i=1}^{\ell(\lambda)} \prod_{j=1}^{\lambda_{i}}\left(\alpha\left(\lambda_{i}-j\right)+\lambda_{j}^{\prime}-i+t\right)
$$

which will be used later on. ${ }^{5}$

\footnotetext{
4 Note that other papers use different conventions. The reader is advised to check the conventions with care.

5 Note that $c(\lambda, \alpha, 1)=\prod_{s \in \lambda} h_{*}^{\lambda}(s)$ and $c(\lambda, \alpha, \alpha)=\prod_{s \in \lambda} h_{\lambda}^{*}(s)$ in terms of the notations of [55].
} 
Given a partition $\lambda$, one defines, in the theory of symmetric functions, the monomial symmetric functions $m_{\lambda}(\mathbf{y})$, over $n$ variables $\mathbf{y}=\left\{y_{r}\right\}, r=1, n$ as $m_{\lambda}(\mathbf{y})=\sum_{\sigma} \prod_{r=1}^{n} y_{\sigma(r)}^{\lambda_{i}}$ where the summation is over all non-equivalent permutations of the variables. For example, given a partition (211) of $k=4, m_{(211)}(\mathbf{y})=y_{1}^{2} y_{2} y_{3}+y_{1} y_{2}^{2} y_{3}+y_{1} y_{2} y_{3}^{2}$. Another useful set of symmetric functions, of obvious importance for the calculation of moments, are the power-sums:

$$
p_{\lambda}(\mathbf{y})=\prod_{i=1}^{\ell(\lambda)} \sum_{r=1}^{n} y_{r}^{\lambda_{i}}
$$

which form a basis of the ring of symmetric functions.

Define the following scalar product, which depends on a real parameter $\alpha$ as

$$
<p_{\lambda}, p_{\mu}>=\delta_{\lambda \mu} z_{\lambda} \alpha^{\ell(\lambda)}, \quad z_{\lambda}=1^{q_{1}} 2^{q_{2}} \cdots q_{1} ! q_{2} ! \cdots
$$

where $q_{i}=q_{i}(\lambda)$ is the number of rows in $\lambda$ whose length are equal to $i$. Here and below we suppress the arguments of all symmetric polynomials except when explicitly needed, for example $p_{\lambda}(\mathbf{y}) \rightarrow p_{\lambda}$. The Jack functions $J_{\lambda}^{(\alpha)}$ obey the following properties (i) orthogonality with respect to the above scalar product (ii) fixed coefficient of highest degree in the monomial basis 6

$$
\begin{aligned}
& <J_{\lambda}^{(\alpha)}, J_{\mu}^{(\alpha)}>=c(\lambda, \alpha, 1) c(\lambda, \alpha, \alpha) \delta_{\lambda \mu} \\
& J_{\lambda}^{(\alpha)}=c(\lambda, \alpha, 1) m_{\lambda}+\sum_{\nu<\lambda} u_{\lambda \nu} m_{\nu}
\end{aligned}
$$

We can go from the basis of power sums to the Jack polynomial basis by the following linear transformations:

$$
J_{\lambda}^{(\alpha)}=\sum_{\nu} \theta_{v}^{\lambda}(\alpha) p_{v}, \quad p_{v}=\sum_{\lambda} \gamma_{\nu}^{\lambda}(\alpha) J_{\lambda}^{(\alpha)}
$$

where the coefficients are in general complicated. Note that the $k$-th moment that we are interested in is precisely the Jacobi ensemble average

$$
<\sum_{r=1}^{n} y_{r}^{k}>_{J}=<p_{(k)}(\mathbf{y})>_{J}
$$

where $(k)$ denotes the partition with only one row of length $k$. Hence we need only the coefficient $\gamma_{\nu=(k)}^{\lambda}$. As we now show one can express this coefficient in terms of $\theta_{\nu=(k)}^{\lambda}$. Indeed, one can write in two ways the following scalar product, first as

$$
<J_{\lambda}^{(\alpha)}, p_{\mu}>=\sum_{\nu} \theta_{\nu}^{\lambda}(\alpha)<p_{\nu}, p_{\mu}>=\theta_{\mu}^{\lambda}(\alpha) z_{\mu} \alpha^{\ell(\mu)}
$$

and also as

$$
<J_{\lambda}^{(\alpha)}, p_{\mu}>=\sum_{\tau} \gamma_{\mu}^{\tau}(\alpha)<J_{\lambda}^{(\alpha)}, J_{\tau}^{(\alpha)}>=\gamma_{\mu}^{\lambda}(\alpha) c(\lambda, \alpha, 1) c(\lambda, \alpha, \alpha)
$$

Hence, comparing we obtain

$$
\gamma_{\mu}^{\lambda}(\alpha)=\frac{\theta_{\mu}^{\lambda}(\alpha) z_{\mu} \alpha^{\ell(\mu)}}{c(\lambda, \alpha, 1) c(\lambda, \alpha, \alpha)}
$$

valid for arbitrary partitions $\mu$ and $\lambda$, which we apply to $\mu=(k)$.

$\overline{6}$ see theorems 5.6 and 5.8 in [55] and (i) p 377 (ii) (10.13-10.22) in [54]. 
Now it turns out that $\theta_{(k)}^{\lambda}(\alpha)$ is known to to be equal to:

$$
\theta_{(k)}^{\lambda}(\alpha)=\prod_{s-\{1,1\}}\left(\alpha a_{\lambda}^{\prime}(s)-l_{\lambda}^{\prime}(s)\right)
$$

if $|\lambda|=k$ and zero otherwise, (see p 383 Ex. 1 (b) in [54] and (19) in [56]), where $a_{\lambda}^{\prime}(s)=j-1$ and $l_{\lambda}^{\prime}(s)=i-1$ are respectively called the co-arm and co-leg lengths of the partition $\lambda$, and the product does not include the box $s=(1,1)$.

Positive moments: this leads to the explicit result for the positive $k$-th moment in terms of an average of the Jack polynomial associated to the partition $(k)$ :

$$
\begin{aligned}
& <\sum_{r=1}^{n} y_{r}^{k}>_{J}=<p_{(k)}(\mathbf{y})>_{J}=\sum_{\lambda,|\lambda|=k} \gamma_{(k)}^{\lambda}(\alpha)<J_{\lambda}^{(\alpha)}(\mathbf{y})>_{J} \\
& \gamma_{(k)}^{\lambda}(\alpha)=\frac{k \alpha}{c(\lambda, \alpha, 1) c(\lambda, \alpha, \alpha)} \prod_{s-\{1,1\}}\left(\alpha a_{\lambda}^{\prime}(s)-l_{\lambda}^{\prime}(s)\right)
\end{aligned}
$$

(see Appendix 6 for an alternative rewriting of this formula).

The problem therefore amounts to evaluating the Jacobi average of $J_{\lambda}^{(\alpha)}(\mathbf{y})$. Such averages where evaluated for a general partition $\lambda$, for a differently normalized set of Jack polynomials, denoted by Macdonald as $P_{\lambda}^{(\alpha)}(\mathbf{y})$, related to the $J_{\lambda}^{(\alpha)}(\mathbf{y})$ as follows

$$
J_{\lambda}^{(\alpha)}(\mathbf{y})=c(\lambda, \alpha, 1) P_{\lambda}^{(\alpha)}(\mathbf{y})
$$

Namely, as was conjectured by Macdonald in [54], and proved by Kadell [52], there exists a closed form expression for $P_{\lambda}^{(\alpha)}(\mathbf{y})$ integrated with the (unnormalized) Jacobi density over the hypercube $\mathbf{y} \in[0,1]^{n}$ with the correspondence

$$
\alpha=1 / \kappa
$$

It is given by

$$
\begin{aligned}
& \int_{[0,1]^{n}} P_{\lambda}^{(1 / \kappa)}(\mathbf{y})|\Delta(\mathbf{y})|^{2 \kappa} \prod_{i=1}^{n} y_{i}^{a}\left(1-y_{i}\right)^{b} d y_{i} \\
& =n ! v_{\lambda}(\kappa) \prod_{i=1}^{n} \frac{\Gamma\left(\lambda_{i}+a+1+\kappa(n-i)\right) \Gamma(b+1+\kappa(n-i))}{\Gamma\left(\lambda_{i}+a+b+2+\kappa(2 n-i-1)\right)}
\end{aligned}
$$

where

$$
v_{\lambda}(\kappa)=\prod_{1 \leq i<j \leq n} \frac{\Gamma\left(\lambda_{i}-\lambda_{j}+\kappa(j-i+1)\right)}{\Gamma\left(\lambda_{i}-\lambda_{j}+\kappa(j-i)\right)}
$$

For the empty partition $\lambda=(0)$ we have $P_{(0)}^{(1 / \kappa)}(\mathbf{y})=1$ and the above integral reduces to the Selberg integral (29).

Recalling the definition of the Jacobi ensemble average (30) and taking the ratio of (74) to (29), we obtain the average of the $P^{(1 / \kappa)}(\mathbf{y})$ polynomial and from it, the average of $J^{(1 / \kappa)}(\mathbf{y})$. The calculation is detailed in the Appendix 5 and the final result is simple and explicit for arbitrary partition $\lambda$

$$
\left\langle J_{\lambda}^{1 / \kappa}(y)\right\rangle_{J}=\kappa^{-|\lambda|} \prod_{i=1}^{\ell(\lambda)} \frac{(a+1+\kappa(n-i))_{\lambda_{i}}}{(a+b+2+\kappa(2 n-i-1))_{\lambda_{i}}}(\kappa(n-i+1))_{\lambda_{i}}
$$


Putting together Eqs. $(70,71)$ and $(76)$ we obtain the $k$-th moment as

$$
\begin{aligned}
\left\langle\frac{1}{n} \sum_{r=1}^{n} y_{r}^{k}\right\rangle_{J}= & \sum_{\lambda,|\lambda|=k} \frac{k \alpha}{c(\lambda, \alpha, 1) c(\lambda, \alpha, \alpha)} \prod_{s-\{1,1\}}\left(\alpha a_{\lambda}^{\prime}(s)-l_{\lambda}^{\prime}(s)\right) \\
& \times \frac{1}{n \kappa^{k}} \prod_{i=1}^{\ell(\lambda)} \frac{(a+1+\kappa(n-i))_{\lambda_{i}}}{(a+b+2+\kappa(2 n-i-1))_{\lambda_{i}}}(\kappa(n-i+1))_{\lambda_{i}}
\end{aligned}
$$

where $\alpha$ should be replaced by $1 / \kappa$ according to (73). Using the explicit expressions for the normalization constants (211) and (212) in Appendix 4 and the above definitions of the co-arm and co-leg, one obtains the formula $(33,34,35)$, for the positive moments given in Sect. 2.2. ${ }^{7}$

We have also used:

$$
\begin{aligned}
\prod_{s-\{1,1\}}\left(\alpha a_{\lambda}^{\prime}(s)-l_{\lambda}^{\prime}(s)\right) & =\alpha^{k-1} \prod_{i=1}^{\ell(\lambda)} \prod_{j=1}^{\lambda_{i}{ }^{\prime}}(j-1-\kappa(i-1)) \\
& =\alpha^{k-1}\left(\lambda_{1}-1\right) ! \prod_{i=2}^{\ell(\lambda)}(-\kappa(i-1))_{\lambda_{i}}
\end{aligned}
$$

where the prime indicates that $i=j=1$ is excluded from the product.

Equivalently, we can rewrite the expression (77) for the moments in a "geometric" form which involves only products over boxes in the Young diagrams, see $(91,92)$ below.

Negative moments: Negative moments can be obtained by applying (70) to the inverse variable, here before averaging (with $k \geq 0$ )

$$
\sum_{r=1}^{n} y_{r}^{-k}=\sum_{\lambda,|\lambda|=k} \gamma_{(k)}^{\lambda}(\alpha) J_{\lambda}^{(\alpha)}\left(\frac{1}{\mathbf{y}}\right)
$$

where we denote $\left(\frac{1}{\mathbf{y}}\right) \equiv\left(\frac{1}{y_{1}}, \cdots \frac{1}{y_{n}}\right)$. We now use the following relation between Jack polynomials, for $n \geq \ell(\lambda)$

$$
P_{\lambda}^{(\alpha)}\left(\frac{1}{\mathbf{y}}\right)=y_{1}^{-l} \cdots y_{n}^{-l} P_{\left(l^{n}-\lambda\right)^{+}}^{(\alpha)}(\mathbf{y})
$$

see [30] p. 643, where $l$ is (a priori) any integer $l \geq \lambda_{1}$ and one denotes

$$
\left.\left(l^{n}-\lambda\right)^{+}=\left\{l, \ldots, l-\lambda_{1}, \ldots, l-\lambda_{\ell(\lambda}\right)\right\}
$$

the partition of length $n$. Using (72) in (80), inserting (81), using again (72), one can now average over the Jacobi measure as follows

$$
\left\langle\sum_{r=1}^{n} y_{r}^{-k}\right\rangle_{J}=\sum_{\lambda,|\lambda|=k} \gamma_{(k)}^{\lambda}(\alpha) \frac{c(\lambda, \alpha, 1)}{c\left(\left(l^{n}-\lambda\right)^{+}, \alpha, 1\right)}\left\langle J_{\left(l^{n}-\lambda\right)^{+}}^{(\alpha)}(\mathbf{y})\right\rangle_{J, a \rightarrow a-l} \frac{S l_{n}(\kappa, a-l, b)}{S l_{n}(\kappa, a, b)}
$$

where the average on the right is over a shifted Jacobi measure with parameter $a-l, b, \kappa$ to account for the prefactor in (81). For the same reason the ratio of Selberg integrals appear, since it is the normalization of the Jacobi measure. We can now use the explicit expressions

7 Note that a textbook treatment of the material discussed in this section is given in [30]. The formula for the positive moments does not appear there explicitly, but can be recovered by integrating (12.145) using (12.143). 
(29) and (76). One finds, after a tedious calculation, similar in spirit to the one described above for the positive moments, the formula (33), (34) for the negative moments given in Sect. 2.2 with

$$
a_{\lambda}^{-}=\prod_{i=1}^{\ell(\lambda)} \frac{(a-l+1+\kappa(i-1))_{l-\lambda_{i}}}{(a-l+b+2+\kappa(n+i-2))_{l-\lambda_{i}}} \frac{(a-l+b+2+\kappa(n+i-2))_{l}}{(a-l+1+\kappa(i-1))_{l}}
$$

where a priori $l$ is an integer sufficiently large. In practice we found that for generic values of $\kappa$ the final result is independent of the choice of $l$ (for each partition), hence we chose $l=0$ which leads to the simplest expression (36) given in in Sect. 2.2.

We now discuss an interesting alternative and useful representation for the (positive and negative) integer moments in terms of contour integrals.

\subsection{Borodin-Gorin Contour Integral Representation of the Moments}

\subsubsection{Positive Moments}

Recently Borodin and Gorin proved integral representations for the moments of the Jacobi $\beta$-ensemble. We refer to the Appendix 1 for derivation and present here a summary of results in our notation. They call these moments $\frac{1}{N} M_{k}(\theta, N, M, \alpha)$ and the correspondence from their four parameters to ours is:

$$
\theta \rightarrow-\beta^{2} \quad N \rightarrow n \quad \theta \alpha-1 \rightarrow a, \quad \theta(M-N+1)-1 \rightarrow b
$$

which leads to the correspondence

$$
<y^{k}>_{\beta, a, b, n}=\frac{1}{n} M_{k}\left(-\beta^{2}, n, n-1-\frac{1+b}{\beta^{2}},-\frac{1+a}{\beta^{2}}\right)
$$

Translated in our parameters their moment formula for positive integer $n$ reads:

$$
\begin{aligned}
<y^{k}>_{\beta, a, b, n}= & \frac{1}{n \beta^{2}} \int \prod_{i=1}^{k} \frac{d u_{i}}{2 i \pi} \prod_{1 \leq i<j \leq k} \frac{u_{j}-u_{i}}{\left(u_{j}-u_{i}+\beta^{2}\right)\left(u_{j}-u_{i}+1\right)} \\
& \times \prod_{1 \leq i+1<j \leq k}\left(u_{j}-u_{i}+1+\beta^{2}\right) \prod_{i=1}^{k} \frac{u_{i}+\beta^{2}}{u_{i}+\beta^{2}(1-n)} \\
& \times \frac{u_{i}-1-a}{u_{i}-2-a-b-\beta^{2}(1-n)}
\end{aligned}
$$

which must be supplemented with the conditions, let us call them $C_{1}$ : $\left|u_{1}\right| \ll\left|u_{2}\right| \ll$ $\left|u_{3}\right| \cdots \ll\left|u_{k}\right|$ and $C_{2}$ : all the contours enclose the singularities at $u_{i}=-(1-n) b^{2}$ and not at $u_{i}=2+a+b+\beta^{2}(1-n)$. These conditions imply that one can first perform the integral on $u_{1}$ and only around the pole $u_{1}=-(1-n) \beta^{2}$ and then iteratively on $u_{2}, u_{3}, \ldots$ and so on.

We will investigate below the properties of this representation in the context of the models we study here. 


\subsubsection{Negative Moments}

Remarkably, Borodin and Gorin also proved a contour integral formula for negative moments, whenever they exist. In our notations, for $k \geq 1$, it reads

$$
\begin{aligned}
& <y^{-k}>_{\beta, a, b, n}=\frac{-1}{n \beta^{2}} \int \prod_{i=1}^{k} \frac{d u_{i}}{2 i \pi} \prod_{1 \leq i<j \leq k} \frac{u_{j}-u_{i}}{\left(u_{j}-u_{i}-\beta^{2}\right)\left(u_{j}-u_{i}-1\right)} \\
& \times \prod_{1 \leq i+1<j \leq k}\left(u_{j}-u_{i}-1-\beta^{2}\right) \prod_{i=1}^{k} \frac{u_{i}-n \beta^{2}}{u_{i}} \times \frac{u_{i}-1-a-b-\beta^{2}(1-n)}{u_{i}-a}
\end{aligned}
$$

which must be supplemented with the conditions, (i) $C_{1}$ : $\left|u_{1}\right| \ll\left|u_{2}\right| \ll\left|u_{3}\right| \ll\left|u_{k}\right|$ and (ii) $C_{2}$ : all the contours enclose the singularities at 0 and not at $a$.

\subsection{Duality}

Let us now discuss an important property of these moment, the duality.

\subsubsection{Statement of the Duality on the Moments}

For clarity let us first recall the property of duality-invariance unveiled in [12]. Consider first a thermodynamic quantity $O_{\beta}$ obtained in the replica formalism in the limit $n=0$. This quantity is said to be duality-invariant if it is well defined in the high temperature region $\beta<1$ and its temperature dependence is given by a function $f(\beta)$ which is known analytically, and satifies $f\left(\beta^{\prime}=1 / \beta\right)=f(\beta)$. The simplest example of such quantities is the mean free energy for Gaussian log-correlated models for which $f(x)=x+1 / x$. More complicated examples are presented in $[12]$, see e.g. $(13,14)$ there. Note that it does not imply anything on the behaviour of $O_{\beta}$ for $\beta>1$, hence it is strictly a property of the high temperature phase. However the property of duality invariance is not restricted to the replica limit $n=0$. In [12] by considering the generating functions for the moments of the partition function, one notices that duality invariance can be extended to finite $n$ by further requiring $n^{\prime} \beta^{\prime}=n \beta$ (see (25) there where $s$ should be identified as $-n \beta$ ).

In the present model, there are more parameters and we can formulate the dualityinvariance property for the moments as follows:

\section{Duality-invariance property}

$$
<y^{k}>_{\beta, a, b, n}=<y^{k}>_{\beta^{\prime}, a^{\prime}, b^{\prime}, n^{\prime}} \quad \beta^{\prime}=1 / \beta \quad n^{\prime}=\beta^{2} n \quad a^{\prime}=a / \beta^{2} \quad b^{\prime}=b / \beta^{2}
$$

which, again, should be understood in the sense of analytical continuation (i.e. it does not provide a mapping from the high to low temperature region as discussed above).

\subsubsection{Checking and Proving Duality for Moments}

The duality property (90) can be checked on the explicit formula (33-35), derived in this paper. Here we focus on positive moments, but similar considerations hold for negative ones, when they exist. To see it explicitly it is convenient to rewrite that formula in a "geometric" form involving only products over boxes in the Young diagrams, as 


$$
<y^{k}>_{\beta, a, b, n}:=\left.\left\langle\frac{1}{n} \sum_{r=1}^{n} y_{r}^{k}\right\rangle_{J}\right|_{\kappa=-\beta^{2}}=\left.\sum_{\lambda,|\lambda|=k} \lim _{\epsilon \rightarrow 0} \frac{k}{n \epsilon} \prod_{s=(i, j) \in \lambda} B_{\lambda}^{\epsilon}(s)\right|_{\kappa=-\beta^{2}}
$$

where

$$
B_{\lambda}^{\epsilon}(s)=\frac{(j-1-(i-1) \kappa+\epsilon)(a+\kappa(n-i)+j)(\kappa(n-i+1)+j-1)}{\left(a_{\lambda}(s)+l_{\lambda}(s) \kappa+1\right)\left(a_{\lambda}(s)+l_{\lambda}(s) \kappa+\kappa\right)(a+b+1+\kappa(2 n-i-1)+j)}
$$

where $\epsilon$ has been introduced only to remove box $(1,1)$ from one of the products and the limit $\epsilon \rightarrow 0$ is trivial. For application to the present purpose we need to set $\kappa=-\beta^{2}$.

The duality is easy to check on that formula and corresponds to the exchange of the partition $\lambda$ with its dual $\lambda^{\prime}$. Indeed it is easy to check that

$$
\left.\left.B_{\lambda}^{\epsilon}(s)\right|_{\kappa=-\beta^{2}}\right|_{\beta, n, a, b}=\left.\left.B_{\lambda^{\prime}}^{\epsilon}\left(s^{\prime}\right)\right|_{\kappa=-\beta^{\prime 2}}\right|_{\beta^{\prime}, n^{\prime}, a^{\prime}, b^{\prime}}
$$

where $s^{\prime}=(j, i)$ is the box in the dual diagram conjugate to $s=(i, j)$, which implies that arm lengths and leg lengths are also exchanged under duality. A similar observation over duality-invariant sum over partitions was reported very recently in [14] for a related problem, about the value at the minimum of a log-correlated field.

Another proof of the duality invariance property for the moments was provided in the recent work Borodin and Gorin (BG). They proved that these moments are rational functions of their four arguments, and that the corresponding analytical continuation in these arguments satisfies an invariance property, which is equivalent to the above duality-invariance (90) under the correspondence (86).

\subsubsection{Consequence of the Duality-Invariance: Freezing}

Let us now examine the implications of the relation (90) for the three examples studied in this paper, showing that a freezing transition at $\beta=1$ is expected in all cases.

For the GUE problem $a=b=\frac{q+\beta^{2}}{2}$ and one finds that the duality invariance in terms of the parameter $q$ can be written as:

$$
q^{\prime}=1+\frac{q-1}{\beta^{2}}
$$

The choice $q=1$ thus ensures duality-invariance of the moments for arbitrary $\beta<1$ and again implies freezing at $\beta=1$.

More generally, starting from Jacobi ensemble measure (28) one can ask how to choose $a$ and $b$ so that the model exhibits the duality-invariance. Consider two (otherwise arbitrary) duality-invariant functions of $\beta, \bar{a}(\beta)$ and $\bar{b}(\beta)$, i.e. satisfying $\bar{a}(1 / \beta)=\bar{a}(\beta)$ and $\bar{b}(1 / \beta)=$ $\bar{b}(\beta)$, and choose:

$$
a=\beta \bar{a}(\beta), \quad b=\beta \bar{b}(\beta)
$$

Then the moments are self-dual. Our second example Eqs. (13) and (14) of a log-correlated potential in presence of a background potential, corresponds to the case of temperature independent constants $\bar{a}$ and $\bar{b}$ and its moments are thus duality-invariant.

Finally, for the $\mathrm{fBm} 0$ the parameter $a=2 n \beta^{2}$ and $b=0$. One checks from (90) that the $\mathrm{fBm} 0$ satisfies duality invariance for arbitrary $n$. In the replica limit $n=0$ we must set 
$a=b=0$, which is a self-dual point, hence for this model the moments obey the following duality-invariance for $\beta<1$

$$
<y^{k}>_{\beta}=<y^{k}>_{1 / \beta}
$$

so that according to the FDC, one should expect them to exhibit freezing at $\beta=1$.

\section{5 $n=0$ Limit of the Moments Formula}

The moment formula obtained above, as well as their contour integral representation are explicit enough to allow for analytic continuation to $n=0$.

\subsubsection{Replica limit of sums over partitions.}

Consider the formula $(33,34)$ inserting $\kappa=-\beta^{2}$. The limit $n \rightarrow 0$ is straightforward, except for one of the factors in the second line for which we use:

$$
\left(-\beta^{2} n\right)_{\lambda_{1}}=-\beta^{2} n\left(1-\beta^{2} n\right)_{\lambda_{1}-1} \simeq_{n \rightarrow 0}-\beta^{2} n\left(\lambda_{1}-1\right) !
$$

This leads to the following formula for the disorder averages of the moments (48) of the general scaled disordered statistical mechanics model (47)

$$
\overline{<y^{k}>_{\beta, a, b}}=\sum_{\lambda,|\lambda|=r} C_{\lambda}^{1} C_{\lambda}^{2}
$$

with

$$
\begin{aligned}
C_{\lambda}^{1}= & -\beta^{2} k\left[\left(\lambda_{1}-1\right) !\right]^{2} \prod_{i=2}^{\ell(\lambda)}\left[\left(\beta^{2}(i-1)\right) \lambda_{i}\right]^{2} \prod_{i=1}^{\ell(\lambda)} \frac{\left(a+1+\beta^{2} i\right) \lambda_{i}}{\left(a+b+2+\beta^{2}(i+1)\right)_{\lambda_{i}}} \\
& \times \prod_{1 \leq i<j \leq \ell(\lambda)} \frac{\lambda_{i}-\lambda_{j}+\beta^{2}(i-j)}{\beta^{2}(i-j)} \\
C_{\lambda}^{2}= & \prod_{i=1}^{\ell(\lambda)} \frac{1}{\left(1+\beta^{2}(i-\ell(\lambda))\right)_{\lambda_{i}}\left(\beta^{2}(i-\ell(\lambda)-1)\right)_{\lambda_{i}}} \prod_{1 \leq i<j \leq \ell(\lambda)} \frac{\left(\beta^{2}(i-j-1)\right) \lambda_{i}-\lambda_{j}}{\left(1+\beta^{2}(i+1-j)\right)_{\lambda_{i}-\lambda_{j}}}
\end{aligned}
$$

This formula is valid in the higher temperature phase of the model, $\beta<1$, where all the factors are clearly finite and non-zero. We will study explicitly below a few moments and their temperature dependence. Note that in the limit $\beta \rightarrow 0$ one recovers the moments (37) (i.e. with the weight $\left.P^{0}(y)\right)$.

The moments of the position of the scaled minimum of the potential as discussed above are recovered in the zero temperature limit $\beta=+\infty$ of the statistical mechanics model. According to the FDC (see section) these moments are equal to their value at the freezing transition $\beta=1$. Hence they can be obtained by taking as limits

$$
\overline{\left(y_{m}\right)^{k}}=\lim _{\beta \rightarrow 1^{-}} \overline{<y^{k}>_{\beta, a, b}}
$$

of the above expression (98-100). However in this expression one easily sees that the factor $C_{2}^{\lambda}$ has poles for $\beta=1$, while $C_{1}^{\lambda}$ is regular and has a finite limit. Examination of low moments, detailed below, show massive cancellations of these poles leading to a well defined finite 
limit. As shown below, using the contour integral representation, this limit is indeed finite for any moment. Note that the poles in the limit $\beta \rightarrow 1$ are also present for $n>0$, so consideration of finite $n$ does not help to handle these cancellations.

The formula (101) together with formula (98-100) thus gives arbitrary positive integer moments of the position of the global minimum of the log-correlated process and as such is a main result of our paper. They can be used to generate these moments to a very high degree on the computer.

\subsubsection{Contour Integral Representation of Moments for $n=0$.}

(i) positive moments To take the limit $n=0$ in the contour integral formula (87) we rewrite

$$
\frac{1}{n \beta^{2}} \prod_{i=1}^{k} \frac{u_{i}+\beta^{2}}{u_{i}+\beta^{2}(1-n)}=\frac{1}{n \beta^{2}} \prod_{i=1}^{k}\left(1+\frac{n \beta^{2}}{u_{i}+\beta^{2}(1-n)}\right)=\frac{1}{n \beta^{2}}+\sum_{i=1}^{k} \frac{1}{u_{i}+\beta^{2}}+O(n)
$$

Inserting the first term in the contour integral gives zero. Next, it is easy to see that only the pole in $u_{1}$ gives non zero residue. Hence we can insert only this term in the integral (87), where we can now safely take $n=0$ leading to the following representation for the disorder average:

$$
\begin{aligned}
\overline{<y^{k}>\beta, a, b}= & \int \prod_{i=1}^{k} \frac{d u_{i}}{2 i \pi} \prod_{1 \leq i<j \leq k} \frac{u_{j}-u_{i}}{\left(u_{j}-u_{i}+\beta^{2}\right)\left(u_{j}-u_{i}+1\right)} \\
& \times \prod_{1 \leq i+1<j \leq k}\left(u_{j}-u_{i}+1+\beta^{2}\right) \frac{1}{u_{1}} \times \prod_{i=1}^{k} \frac{u_{i}-1-a-\beta^{2}}{u_{i}-2-a-b-2 \beta^{2}}
\end{aligned}
$$

where we have shifted $u_{i} \rightarrow u_{i}-\beta^{2}$ for convenience. This again must be supplemented with the condition (i) $C_{1}:\left|u_{1}\right| \ll\left|u_{2}\right| \ll\left|u_{3}\right| \cdots \ll\left|u_{p}\right|$ and (ii) $C_{2}$ : all the contours enclose the singularities at $u_{1}=0$ but not at $u_{i}=2+a+b+2 \beta^{2}$. In practice the contours will run (and close) in the negative half plane $\operatorname{Re}\left(u_{i}\right)<0$ and pick up residues from poles on the negative real line. We note that one needs the condition $2+a+b+2 \beta^{2}>0$ which, for $a=b$ is precisely the one found in [12] corresponding to a binding transition to the edge (for $a<-1-\beta^{2}$ ). We will thus assume that the condition is fulfilled, which is the case for all three examples considered here.

The limit $\beta \rightarrow 1$ can be performed explicitly leading to a contour integral representation for the positive integer moments of the position of the global extremum of the log-correlated field

$$
\overline{y_{m}^{k}}=\int \prod_{i=1}^{k} \frac{d u_{i}}{2 i \pi} \prod_{1 \leq i<j \leq k} \frac{u_{j}-u_{i}}{\left(u_{j}-u_{i}+1\right)^{2}} \prod_{1 \leq i+1<j \leq k}\left(u_{j}-u_{i}+2\right) \frac{1}{u_{1}} \prod_{i=1}^{k} \frac{u_{i}-2-a}{u_{i}-4-a-b}
$$

with the same contour conditions $C_{1}$ and $C_{2}$. This formula should thus be equivalent to our main result (98-101), which we have checked for a few low order moments.

(i) negative moments The same manipulation as above in the limit $n \rightarrow 0$ gives the disorder averaged moments for $k \geq 1$ : 


$$
\begin{aligned}
\overline{<y^{-k}>_{\beta, a, b}}= & \int \prod_{i=1}^{k} \frac{d u_{i}}{2 i \pi} \prod_{1 \leq i<j \leq k} \frac{u_{j}-u_{i}}{\left(u_{j}-u_{i}-\beta^{2}\right)\left(u_{j}-u_{i}-1\right)} \\
& \times \prod_{1 \leq i+1<j \leq k}\left(u_{j}-u_{i}-1-\beta^{2}\right) \frac{1}{u_{1}} \times \prod_{i=1}^{k} \frac{u_{i}-1-a-b-\beta^{2}}{u_{i}-a}
\end{aligned}
$$

provided these moment exist. Taking again the limit $\beta \rightarrow 1$ one obtains the negative integer moments of the position of the global extremum of the log-correlated field as

$$
\begin{aligned}
\overline{y_{m}^{-k}}= & \int \prod_{i=1}^{k} \frac{d u_{i}}{2 i \pi} \prod_{1 \leq i<j \leq k} \frac{u_{j}-u_{i}}{\left(u_{j}-u_{i}-1\right)^{2}} \prod_{1 \leq i+1<j \leq k}\left(u_{j}-u_{i}-2\right) \frac{1}{u_{1}} \\
& \times \prod_{i=1}^{k} \frac{u_{i}-2-a-b}{u_{i}-a}
\end{aligned}
$$

for $k$ positive integer, provided they exist. In both integrals the contours obey the two conditions (i) $C_{1}:\left|u_{1}\right| \ll\left|u_{2}\right| \ll\left|u_{3}\right| \cdots \ll\left|u_{k}\right|$ and (ii) $C_{2}$ : all the contours enclose the singularities at 0 and not at $a$. At present there is no equivalent formula in terms of sums over partitions, hence the above formula is an important result of the paper.

We now turn to explicit study of the low moments

\subsection{Calculation and Results for the First Moment}

Let us illustrate the calculation using the contour integral (87) on the simplest example of the first moment $k=1$

$$
\begin{aligned}
<y>_{\beta, a, b, n} & =\frac{1}{n \beta^{2}} \int \frac{d u_{1}}{2 i \pi} \frac{u_{1}+\beta^{2}}{u_{1}+\beta^{2}(1-n)} \frac{u_{1}-1-a}{u_{1}-2-a-b-\beta^{2}(1-n)} \\
& =\frac{1+a-\beta^{2}(n-1)}{2+a+b-2 \beta^{2}(n-1)}
\end{aligned}
$$

which is equal to the residue at $u_{1}=-(1-n) \beta^{2}$. In terms of partitions only the partition $\lambda=(1)$ contributes, so it is easy to see on $(33,34)$, and even more immediate on $(91,92)$ [using $i=j=1, a_{\lambda}=\ell_{\lambda}=0$ ], that it reproduces (71). One can explicitly verify on this result that the first moment is invariant by the duality transformation (90).

The $n=0$ limit yields the disorder averaged first moment

$$
\overline{<y>\beta, a, b}=\frac{1+a+\beta^{2}}{2+a+b+2 \beta^{2}}
$$

For the symmetric situation $a=b$, which is the case both for the $\operatorname{fBm} a=b=0$ and for the GUE characteristic polynomial $a=b=\frac{1+\beta^{2}}{2}$ the first moment is thus simply

$$
\overline{<y>_{\beta}}=\frac{1}{2}
$$

In the second example of the LCGP with edge charges one obtains:

$$
\overline{<y>_{\beta}}=\frac{1+\bar{a} \beta+\beta^{2}}{2+(\bar{a}+\bar{b}) \beta+2 \beta^{2}}
$$


The duality-freezing conjecture then leads to the the first moment of the position of the minimum

$$
\overline{y_{m}}=\frac{2+\bar{a}}{4+\bar{a}+\bar{b}}, \quad \overline{y_{m}}-\frac{1}{2}=\frac{\bar{a}-\bar{b}}{2(\bar{a}+\bar{b}+4)}
$$

which is Eq. (15) in Prediction 2.

These results can be compared to the first moment in absence of the random potential and at finite inverse temperature $\beta$, i.e. from the measure $\left.P^{0}(y)\right|_{a=\beta \bar{a}, b=\beta \bar{b}}$

$$
<y>_{P^{0}}-\frac{1}{2}=\frac{\beta(\bar{a}-\bar{b})}{2(2+\beta(\bar{a}+\bar{b}))}
$$

which reproduces the absolute minimum $y_{m}^{0}=\bar{a} /(\bar{a}+\bar{b})$ in the absence of disorder for $\beta=+\infty$. Comparing with (111) shows that even at the freezing temperature $\beta=1$, disorder brings the average position closer to the midpoint $y=\frac{1}{2}$.

\subsection{Results for Second, Third and Fourth Moments}

The calculation of the second moment by the contour integral method is relatively simple, and sketched in the Appendix 2 Sect. 1 for $n=0$. It leads to the disorder average:

$$
\overline{<y^{2}>_{\beta, a, b}}=\frac{\left(a+\beta^{2}+1\right)\left(\beta^{2}(4 a+2 b+9)+(a+2)(a+b+2)+4 \beta^{4}\right)}{\left(a+2 \beta^{2}+b+2\right)\left(a+2 \beta^{2}+b+3\right)\left(a+3 \beta^{2}+b+2\right)}
$$

This expression is also easily recovered from the sum $(33,34)$, or $(91,92)$ involving now the two partitions (2) and $(1,1)$. For $\beta=0$ it reproduces (37), i.e. the trivial average with respect to the weight $P^{0}(y) \sim y^{a}(1-y)^{b}$. The expression (113) is duality-invariant and we expect that it freezes at $\beta=1$ leading to the predictions for the second moment of the position of the extremum.

Let us now detail the results for each example separately, including moments up to $k=4$ when space permits, more detailed derivations and results being displayed in the Appendix 2.

\subsubsection{Log-Correlated Potential with Edge Charges $\bar{a}, \bar{b}$.}

The second moment of the position of the global minimum is obtained from above as ${ }^{8}$

$$
\overline{y_{m}^{2}}=\frac{(\bar{a}+2)(\bar{a}(\bar{a}+\bar{b}+8)+4 \bar{b}+17)}{(\bar{a}+\bar{b}+4)(\bar{a}+\bar{b}+5)^{2}}
$$

This leads to the variance (16) in Prediction 2, replacing there $x \rightarrow y$. Expressions for higher moments for general $\bar{a}, \bar{b}$ are too bulky to present here, and we only display the third moment in (192) and the skewness in (193).

Two special cases are of interest:

(i) only one edge charge, at $y=0$ : One sets $\bar{b}=0$. Let us give here the skewness in that case

$$
S k:=\frac{\overline{\left(y_{m}-\overline{y_{m}}\right)^{3}}}{\frac{\overline{\left.y_{m}-\overline{y_{m}}\right)^{2}}}{2}}=-\frac{\bar{a}(\bar{a}+5)(\bar{a}(7 \bar{a}+68)+164)}{\sqrt{2} \sqrt{\bar{a}+2}(\bar{a}+6)^{2}(2 \bar{a}+9)^{3 / 2}}
$$

8 One sees on this expression that binding to the edge $y_{m} \rightarrow 0^{+}$(resp. $y_{m} \rightarrow 1^{-}$) occurs when $a \rightarrow-2^{+}$ (resp. $\bar{b} \rightarrow-2$ ) so one may surmise that the result is valid as long as $-2<\bar{a}, \bar{b}$. 
which is negative. It can be compared with the skewness associated to the measure $\sim y^{\bar{a}}$, which is

$$
S k_{0}=-\frac{2 \bar{a} \sqrt{\bar{a}+3}}{\sqrt{\bar{a}+1}(\bar{a}+4)}
$$

One finds that $S k$ decreases from 0 to $-7 / 4$ as $\bar{a}$ increases, while $S k_{0}$ decreases from 0 to -2 , hence they are quite distinct.

(ii) symmetric case $\bar{a}=\bar{b}$. The variance is obtained as:

$$
\overline{y_{m}^{2}}-{\overline{y_{m}}}^{2}=\frac{4 \bar{a}+9}{4(2 \bar{a}+5)^{2}}
$$

where we recall, $\overline{y_{m}}=\frac{1}{2}$, and we checked explicity that the moment formulae lead to

$$
\overline{\left(y_{m}-\frac{1}{2}\right)^{3}}=0
$$

as expected by symmetry $y \rightarrow 1-y$. The fourth moment and kurtosis are obtained as

$$
\begin{aligned}
& \overline{y_{m}^{4}}=\frac{4 a^{5}+84 a^{4}+663 a^{3}+2488 a^{2}+4478 a+3110}{4(a+3)(2 a+5)^{2}(2 a+7)^{2}} \\
& \mathrm{Ku}=-\frac{2\left(8 a^{5}+248 a^{4}+2054 a^{3}+7328 a^{2}+12053 a+7523\right)}{(a+3)(2 a+7)^{2}(4 a+9)^{2}}
\end{aligned}
$$

The kurtosis can be compared to the one of the measure $y^{\bar{a}}(1-y)^{\bar{a}}$ which is:

$$
\kappa_{0}=-\frac{6}{5+2 a}
$$

Note that when $\bar{a}$ increases, $\mathrm{Ku} \rightarrow-1 / 4$ while $\mathrm{Ku}_{0} \rightarrow 0$.

\subsubsection{GUE Characteristic Polynomial}

For the GUE-CP we must insert $a=b=\frac{1+\beta^{2}}{2}$, in (92). The second moment for the associated statistical mechanics model in the high temperature phase $\beta<1$, and for the position of the global minimum (obtained by setting $\beta=1$ ) are then found to be:

$$
\overline{<y^{2}>_{\beta}}=\frac{15 \beta^{4}+32 \beta^{2}+15}{4\left(3 \beta^{2}+4\right)\left(4 \beta^{2}+3\right)}, \quad \overline{y_{m}^{2}}=\frac{31}{98}
$$

For completeness the expression at finite $n$ is given in (191). In the original variable $x \in$ $[-1,1]$, i.e. the support of the semi-circle, using $x=1-2 y$ and the result for the first moment we obtain:

$$
\overline{<x^{2}>_{\beta}}=\frac{3+7 \beta^{2}+3 \beta^{4}}{\left(4+3 \beta^{2}\right)\left(3+4 \beta^{2}\right)}, \quad \overline{x_{m}^{2}}=\frac{13}{49}=0.265306 \ldots
$$

where we expect now the PDF of the position of the maximum, $\mathcal{P}(x)$, to be centered and symmetric around $x=0$ (which was checked explicitly up to fifth moment).

We give directly the fourth moment of the position of the maximum (see 1 for finite temperature expressions)

$$
\overline{y_{m}^{4}}=\frac{401}{2352}, \quad \overline{x_{m}^{4}}=\frac{20}{147}=0.136054 \ldots
$$


which leads to the fourth cumulant and kurtosis as:

$$
{\overline{x_{m}^{4}}}^{c}=-\frac{541}{7203}, \quad \mathrm{Ku}=-\frac{541}{507}=-1.06706 \ldots
$$

These moments can be compared with the ones of the semi-circle density

$$
<x^{k}>_{\rho}:=\int_{-1}^{1} d x x^{k} \rho(x), \quad \rho(x)=\frac{2}{\pi} \sqrt{1-x^{2}}
$$

which are:

$$
<x^{2}>_{\rho}=\frac{1}{4}, \quad<x^{4}>_{\rho}=\frac{1}{8}=0.125, \quad \mathrm{Ku}=-1
$$

and are found quite close, suggesting that $\mathcal{P}(x)$ is distinct from, but numerically close, to the semi-circle density.

\subsubsection{Fractional Brownian Motion}

For the fBm0 we should set $a=b=0$ in (113) leading to the following disorder average of the associated statistical mechanics model in the high temperature phase:

$$
\overline{<y^{2}>_{\beta}}=\frac{4 \beta^{4}+9 \beta^{2}+4}{2\left(2 \beta^{2}+3\right)\left(3 \beta^{2}+2\right)}, \quad \overline{<y^{2}>_{\beta}}-\overline{<y>_{\beta}^{2}}=\frac{\left(\beta^{2}+2\right)\left(2 \beta^{2}+1\right)}{4\left(2 \beta^{2}+3\right)\left(3 \beta^{2}+2\right)}
$$

which is manifestly duality-invariant (see (190) for the $n$-dependence). The second moment of the position of the global minimum of the $\mathrm{fBm} 0$ is thus predicted to be

$$
\overline{y_{m}^{2}}=\frac{17}{50}, \quad \overline{y_{m}^{2}}-{\overline{y_{m}}}^{2}=\frac{9}{100}
$$

as displayed in Prediction 3. This is distinct, but numerically not very different, from what is obtained from a uniform distribution $P^{0}(y)=1$ on $[0,1]$, namely $<y^{2}>_{P^{0}}=\frac{1}{3}$ and $<\left(y-\frac{1}{2}\right)^{2}>_{P^{0}}=\frac{1}{12}$.

All odd moments centered around $y=\frac{1}{2}$ are predicted to vanish, as in (118). Although we explicitly checked up to fifth it should be a general property. The asymmetry induced by fixing one point of the $\mathrm{fBm} 0$ at $x=0$ and letting the one at $x=1$ free, does not manifest itself in the moments of the minimum (or at any temperature in the statistical mechanics model). It does arise however to first order in $n$ (as seen e.g. from (71)) and is detectable in the joint distribution of values and positions of the minimum (see below).

From the formula (119) specialized to $a=0$ we obtain respectively the fourth moment, the fourth cumulant and the kurtosis $\kappa$ for the position of the minimum of the fBm0:

$$
\begin{gathered}
\overline{y_{m}^{4}}=\frac{311}{1470}, \quad{\overline{y_{m}^{4}}}^{c}: \overline{\left(y_{m}-\frac{1}{2}\right)^{4}}-3 \overline{\left(y_{m}-\frac{1}{2}\right)^{2}}=\frac{-7523}{735000} \\
\kappa:=\frac{{\overline{y_{m}^{4}}}^{c}}{{\overline{y_{m}^{2}}}^{2}}=\frac{-15046}{11907}=-1.26363 \ldots
\end{gathered}
$$

These three numbers are respectively $\frac{1}{5},-\frac{1}{120}$ and -1.2 for a uniform distribution of [0.1], hence a difference of a few percent. 


\subsection{Results for Negative Moments}

The negative moments lead interesting additional information on the three problems under study. Let us present the (short) calculation of the first negative moment, and also give the expression for the second. Equation (89) for $k=1$ yields

$$
\begin{aligned}
<y^{-1}>_{\beta, a, b, n} & =\frac{-1}{n \beta^{2}} \int \frac{d u_{1}}{2 i \pi} \frac{u_{1}-n \beta^{2}}{u_{1}} \times \frac{u_{1}-1-a-b-\beta^{2}(1-n)}{u_{1}-a} \\
& =\frac{1+a+b+\beta^{2}(1-n)}{a}
\end{aligned}
$$

The continuation to $n=0$ of this formula, and of the one for the second negative moment (calculated in Appendix 2, Sect. 1 ) leads to the following disorder averages in the statistical mechanics model for $\beta^{2}<1$

$$
\overline{<y^{-1}>_{\beta}}=\frac{1+a+b+\beta^{2}}{a}, \overline{<y^{-2}>_{\beta}}=\frac{\left(a+\beta^{2}+b+1\right)\left(a(a+b)+\beta^{2}\right)}{(a-1) a\left(a-\beta^{2}\right)}
$$

Obviously the same formula exist hold exchanging $y \rightarrow 1-y$ and $a \rightarrow b$. One can check that these formula coincide with the ones obtained from the general result (34-36).

One can check that for $\beta=0$ and $a, b$ fixed, i.e. in the absence of the random potential, these formula agree with the same averages over the deterministic measure $P^{0}(y) \sim y^{a}(1-$ $y)^{b}$, i.e. Eq. (37) setting $k=-1,-2$ there. The effect of the disorder is thus to increase the values of the inverse moments, presumably from the events when favorable regions in the random potential appear near the edges. We see that $a>0$ (repulsive charge at $y=0$ ) is required for the finiteness of the first inverse moment, and $a>1$ for the finiteness of the second. In addition, since $y \in[0,1]$, one must have $\overline{<y^{-1}>_{\beta}} \geq 1$. Hence a binding transition at $y=1$ must occur when the charge becomes too attractive, for $b \leq b_{c}=-1-\beta^{2}$. Symmetric conditions hold under exchanges of $y \rightarrow 1-y$ and $(a, b) \rightarrow(b, a)$.

These moments give some information about the disorder-averaged Gibbs measure: if we assume a power law behavior near the edge, $\overline{p_{\beta}(y)} \sim_{y \rightarrow 0} y^{c}$, the exponent $c=c(a, b, \beta)$ must be such that $c>0$ whenever $a>0$, and $c>1$ whenever $a>1$. From the divergence of the inverse moments when $a$ reaches these values, we can surmise that $c$ also vanishes at $a=0$ and equals 1 at $a=1$. The simplest possible scenario then is that $c=a$, but it remains to be confirmed.

The effect of disorder saturates at $\beta=1$ where we predict freezing in these negative moments, which, as can be checked on (134) using (90) are duality-invariant. As discussed above we predict that the full PDF $\overline{p_{\beta}(y)}$ freezes, i.e. $\mathcal{P}\left(y_{m}\right)=\overline{p_{\beta=1}(y)}$. Let us now discuss consequences for our three examples.

(i) For the GUE-CP, one must set $a=b=\frac{1+\beta^{2}}{2}$. One sees that the first inverse moment exist, but not the second, so the exponent $0<c<1$. One finds that the first inverse moment is temperature independent:

$$
\overline{<y^{-1}>\beta}=4, \quad 0 \leq \beta \leq 1
$$

which leads to the following predictions for the position of the maximum

$$
\overline{y_{m}^{-1}}=4, \overline{\left(1-x_{m}\right)^{-1}}=2
$$


Remarkably, this is also exactly the value of the same average with respect to the semicircle density

$$
<\frac{1}{1-x}>_{\rho}=2
$$

This suggests that the two distributions, $\mathcal{P}(x)$ ands $\rho(x)$, although distinct, are very similar near the edges.

(ii) LCP with edge charges, one must set $a=\beta \bar{a}, b=\beta \bar{b}$. The prediction for the first two inverse moments of the position of the global minimum is:

$$
\overline{y_{m}^{-1}}=\frac{2+\bar{a}+\bar{b}}{\bar{a}}, \quad \overline{y_{m}^{-2}}=\frac{(\bar{a}+\bar{b}+2)(\bar{a}(\bar{a}+\bar{b})+1)}{\bar{a}(\bar{a}-1)^{2}}
$$

where domain of existence has been discussed above. It would be interesting to see whether the simplest scenario for the edge behavior, i.e. that $\mathcal{P}\left(y_{m}\right) \sim y_{m}^{a}$ near $y=0$, and by symmetry $\mathcal{P}\left(y_{m}\right) \sim y_{m}^{b}$ near $y=1$ can be confirmed (or infirmed) in future studies.

(iii) For the $\mathrm{fBmO}$ one must set $a=b=0$ (for $n=0$ ), and neither of these moments exist. Hence the edge exponent of $\mathcal{P}(y) \sim y^{c}$ is such that $c \leq 0$ (and probably $c=0$ ).

\subsection{Correlation Between Position and Value of the Minimum}

Until now we used only the values of the moments at $n=0$. However they do exhibit a non-trivial dependence in the number of replica $n$. One may thus ask what is the information encoded in that dependence.

The detailed analysis is performed in footnote ${ }^{9}$ The answer is that the knowledge of the $n$-dependence of all moments allows in principle to reconstruct the joint distribution, $\mathcal{P}\left(x_{m}, V_{m}\right)$, of the position and value of the extremum. This is an ambitious task which is far from completed. However in footnote 9 we give a general formula for the conditional moments, i.e the moments of $x_{m}$ conditioned to a particular value of $V_{m}$.

Here we display the results for the simplest cross-correlations in the LCGP model with edge charges, the derivation and more results are given in footnote 9 . We find

$$
\begin{aligned}
& \overline{y_{m} V_{m}}-\overline{y_{m}} \overline{V_{m}}=\frac{\bar{b}-\bar{a}}{(\bar{a}+\bar{b}+4)^{2}} \\
& \overline{\left(y_{m}-\overline{y_{m}}\right)\left(V_{m}-\overline{V_{m}}\right)^{2}}=\frac{4(\bar{a}-\bar{b})}{(\bar{a}+\bar{b}+4)^{3}}
\end{aligned}
$$

In the case $\bar{a}=\bar{b}$ these two correlations of the first moment vanish, and so do higher ones: one shows (from the general formula in footnote 9) that the first conditional moment, $\mathbb{E}\left(y_{m} \mid V_{m}\right)=\frac{1}{2}$ independently of $V_{m}$. Continuing with the case $\bar{a}=\bar{b}$ we further obtain

$$
\begin{aligned}
& \overline{\left(y_{m}-\frac{1}{2}\right) V_{m}}=0 \\
& \overline{\left(y_{m}^{2}-\overline{y_{m}^{2}}\right)\left(V_{m}-\overline{V_{m}}\right)}=\frac{(\bar{a}+2)(2 \bar{a}+1)}{2(2 \bar{a}+5)^{3}} \\
& \overline{\left(y_{m}^{2}-\overline{y_{m}^{2}}\right)\left(V_{m}-\overline{V_{m}}\right)^{2}}=-\frac{4 \bar{a}^{2}+8 \bar{a}+1}{(2 \bar{a}+5)^{4}}
\end{aligned}
$$

${ }^{9}$ See Appendix 8 of the ArXiv version of the present paper: http://arxiv.org/abs/1511.04258. 
Setting $\bar{a}=1$ leads to the prediction for the GUE-CP as

$$
\begin{aligned}
& \overline{x_{m}\left(V_{m}-\overline{V_{m}}\right)}=0, \quad \overline{x_{m}\left(V_{m}-\overline{V_{m}}\right)^{2}}=0 \\
& \overline{\left(y_{m}^{2}-\overline{y_{m}^{2}}\right)\left(V_{m}-\overline{V_{m}}\right)}=\frac{9}{686}, \quad \overline{\left(x_{m}^{2}-\overline{x_{m}^{2}}\right)\left(V_{m}-\overline{V_{m}}\right)}=\frac{18}{343} \\
& \overline{\left(x_{m}^{2}-\overline{x_{m}^{2}}\right)\left(V_{m}-\overline{V_{m}}\right)^{2}}=-\frac{52}{2401}
\end{aligned}
$$

and we recall that $x_{m}=1-2 y_{m}$ with $\overline{y_{m}}=\frac{1}{2}$ for the GUE-CP and in fact, as discussed above the first conditional moment $\mathbb{E}\left(x_{m} \mid V_{m}\right)=0$ vanishes for any $V_{m}$.

The $\mathrm{fBm} 0$, as we defined it with the value fixed at $y=0$, provides an interesting example of a process with non-trivial correlation. Indeed the above formula must be modified since $a=2 \beta^{2} n$ for the fBm0. As discussed in Appendix 7, that leads to difficulties in the method for the determining the PDF of $V_{m}$. One should thus be careful in assessing the validity of the following results for the case of the fBm0. They read

$$
\begin{aligned}
& \overline{\left(y_{m}-\overline{y_{m}}\right)\left(V_{m}-\overline{V_{m}}\right)}=-\frac{1}{4} \\
& \overline{\left(y_{m}-\overline{y_{m}}\right)\left(V_{m}-\overline{V_{m}}\right)^{2}}=0 \\
& \overline{\left(y_{m}^{2}-\overline{y_{m}^{2}}\right)\left(V_{m}-\overline{V_{m}}\right)}=-\frac{21}{100} \\
& \overline{\left(y_{m}^{2}-\overline{y_{m}^{2}}\right)\left(V_{m}-\overline{V_{m}}\right)^{2}}=\frac{2}{25}
\end{aligned}
$$

and we recall $\overline{y_{m}}=\frac{1}{2}$ for the $\mathrm{fBm}$. The negative value obtained for the first correlation (first line) is a reflection of the boundary condition chosen, namely pinning of $B_{0}^{(\eta)}(y=0)=0$ and free boundary condition at $y=1$, which allows for lower values of the minimum near the right edge. The vanishing of the second line follows from the discussion in footnote 9.

\section{Other Ensembles}

\subsection{General Considerations}

Once the moments for the Jacobi ensemble are known, one can obtain moments in a few other ensembles. Define the generic measure

$$
\mathcal{P}_{A}(\mathbf{y}) d \mathbf{y}=\frac{1}{\mathcal{Z}_{n}^{A}} \prod_{i=1}^{n} d y_{i} \mu_{A}\left(y_{i}\right) \prod_{1 \leq i<j \leq n}\left|y_{i}-y_{j}\right|^{2 \kappa}
$$

with, for Jacobi, $\mu_{A}(y)=\mu_{J}(y):=y^{a}(1-y)^{b} \theta(0<y<1)$. The main other ensembles differ only by the choice of the weight function $\mu_{A}(y)$.

(i) Laguerre ensemble Define $y_{i}=z_{i} / b$ and take the limit $b \rightarrow+\infty$. Then

$$
\lim _{b \rightarrow+\infty} \mathcal{P}_{J}(\mathbf{y}) d \mathbf{y}=\mathcal{P}_{L}(\mathbf{z}) d \mathbf{z}, \quad \mu_{A}(z)=z^{a} e^{-z} \theta(z)
$$

where $\mathcal{Z}_{n}^{L}=\lim _{b \rightarrow+\infty} b^{a+n+\kappa n(n-1) / 2} \mathcal{Z}_{n}^{J}=\prod_{j=1}^{n} \frac{\Gamma(1+a+(j-1) \kappa) \Gamma(1+j \kappa)}{\Gamma(1+\kappa)}$.

Hence the moments in Laguerre ensemble are obtained as:

$$
<z^{k}>_{L}=\lim _{b \rightarrow \infty} b^{k}<y^{k}>_{J}
$$


However in our statistical mechanics model, for instance the LCG random potential with an external background, we need to define $z$ slightly differently, i.e. $z=\frac{b}{\beta} y=\bar{b} y$. This ensures duality invariance of the problem, and the Laguerre weight can now be interpreted as a bona-fide external background potential:

$$
V_{0}(z)=-\bar{a} \ln z+z
$$

which confines the particle near the edge $z=0$ (for $\bar{a}>0$ ).

(ii) Gaussian-Hermite ensemble Define $y_{i}=\frac{1}{2}+\left(z_{i} / \sqrt{8 a}\right)$ and take the limit $a=b \rightarrow+\infty$. Then

$$
\lim _{a=b \rightarrow+\infty} \mathcal{P}_{J}(\mathbf{y}) d \mathbf{y}=\mathcal{P}_{G}(\mathbf{z}) d \mathbf{z}, \quad \mu_{G}(z)=e^{-z^{2} / 2}
$$

where $\mathcal{Z}_{n}^{G}=\lim _{a=b \rightarrow+\infty} 4^{a n}(8 a)^{n / 2+\kappa n(n-1) / 2} \mathcal{Z}_{n}^{J}=(2 \pi)^{n / 2} \prod_{j=1}^{n} \frac{\Gamma(1+j \kappa)}{\Gamma(1+\kappa)}$ is the Mehta integral. Similarly below we will introduce a factor of $\beta$ in the definition, see next section.

(iii) Inverse-Jacobi weights Define $y_{i}=1 / z_{i}$ Then

$$
\mathcal{P}_{J}(\mathbf{y}) d \mathbf{y}=\mathcal{P}_{L}(\mathbf{z}) d \mathbf{z}, \quad \mu_{A}(z)=z^{-2-a-b-2(n-1) \kappa}(z-1)^{b} \theta(z-1)
$$

where $\mathcal{Z}_{n}^{I}=\mathcal{Z}_{n}^{J}$

There is a second set of models, for which the correspondence is less direct. We will follow the arguments of [39] to surmise a relation between moments. As in that work, one starts with the simple identity, for $k \in \mathbb{Z}, a \in \mathbb{R}$

$$
\int_{-\pi}^{\pi} d \theta e^{i \theta(a+1+k)}=\frac{2 \sin ((1+a+k) \pi)}{1+a+k}=2(-1)^{k} \sin ((1+a) \pi) \int_{0}^{1} d t t^{a+k}
$$

valid whenever the last integral converge. That leads to the multiple-integral version

$$
\prod_{j=1}^{n} \int_{-\pi}^{\pi} d \theta_{j} e^{i \theta_{j}(a+1)} f\left(-e^{i \theta_{1}}, \ldots-e^{i \theta_{n}}\right)=[2 \sin ((1+a) \pi)]^{n} \prod_{j=1}^{n} \int_{0}^{1} d t_{j} t_{j}^{a} f\left(t_{1}, . . t_{n}\right)
$$

for any Laurent polynomial $f$. From this one conjectures interesting relations between quantities in the circular and Jacobi ensembles, see (1.15-1.17) in [39] as well as Proposition 13.1.4 in Chap.13 of [30]. Further elaborations of these relations lead us to the following conjectures for the moments:

(i) Circular ensemble with weight. Consider the CUE with weight, defined by the joint probability

$$
\frac{1}{\mathcal{Z}_{n}^{C}} \prod_{i=1}^{n} \frac{d \theta_{i}}{2 \pi}\left|1+e^{i \theta_{i}}\right|^{2 \mu} \prod_{1 \leq i<j \leq n}\left|e^{i \theta_{i}}-e^{i \theta_{j}}\right|^{2 \kappa}
$$

for the variables $\theta_{i} \in[-\pi, \pi[$. Then we conjecture that

$$
<\cos (k \theta)>_{\text {circular }}=(-1)^{k}<y^{k}>\left._{\kappa, a, b, n}\right|_{a=-\mu-1-\kappa(n-1), b=2 \mu}
$$

(ii) Cauchy- $\beta$ ensemble. Following (1.19) in [39] and using the stereographic projection from the circle to the real axis, $e^{i \theta}=(i-z) /(i+z)$ we obtain the Cauchy- $\beta$ ensemble which has weight on the whole real axis $z \in \mathbb{R}$

$$
\mu_{C}(z)=\frac{1}{\left(1+z^{2}\right)^{\rho}}, \quad \rho=1+\mu+(n-1) \kappa
$$


For this ensemble, the conjecture then becomes

$$
<\operatorname{Re}\left[\left(\frac{i-z}{i+z}\right)^{k}\right]>_{\text {Cauchy }}=(-1)^{k}<y^{k}>\left._{\kappa, a, b, n}\right|_{a=-\rho, b=2 \rho-2-2(n-1) \kappa}
$$

which we checked is obeyed for $\kappa=0$, in which case one has $<y^{k}>_{J, \kappa=0}=$ $(1-\rho)_{k} /(\rho)_{k}$. Note that interesting integrable generalization of Cauchy ensemble was proposed in [57].

Let us now study the two following examples in more details

\subsection{Moments for the Laguerre Ensemble}

\subsubsection{General Formula}

From (33-35), performing the limit (153) we obtain the general positive moments of the Laguerre ensemble as ${ }^{10}$

$$
\left\langle\frac{1}{n} \sum_{j=1}^{n} y_{j}^{k}\right\rangle_{L}=\sum_{\lambda,|\lambda|=k} A_{\lambda} a_{\lambda}^{+}
$$

where the sum is over all partitions of $k$, and

$$
\begin{aligned}
A_{\lambda} a_{\lambda}^{+}= & \frac{k\left(\lambda_{1}-1\right) !}{(\kappa(\ell(\lambda)-1)+1)_{\lambda_{1}}} \prod_{i=2}^{\ell(\lambda)} \frac{(\kappa(1-i))_{\lambda_{i}}}{(\kappa(\ell(\lambda)-i)+1)_{\lambda_{i}}} \prod_{1 \leq i<j \leq \ell(\lambda)} \frac{\kappa(j-i)+\lambda_{i}-\lambda_{j}}{\kappa(j-i)} \\
& \times \frac{1}{n} \prod_{i=1}^{\ell(\lambda)}(a+1+\kappa(n-i))_{\lambda_{i}} \frac{(\kappa(n-i+1))_{\lambda_{i}}}{(\kappa(\ell(\lambda)-i+1))_{\lambda_{i}}} \prod_{1 \leq i<j \leq \ell(\lambda)} \frac{(\kappa(j-i+1))_{\lambda_{i}-\lambda_{j}}}{(\kappa(j-i-1)+1) \lambda_{i}-\lambda_{j}}
\end{aligned}
$$

i.e. a single factor has disappeared. It turns out that these sums are polynomials, although it may not be easy to see on this expression.

Let us give the first two moments:

$$
\begin{aligned}
& <z>_{L}=1+a+\kappa(n-1) \\
& <z^{2}>_{L}=(1+a+\kappa(n-1))(2+a+2 \kappa(n-1))
\end{aligned}
$$

higher moments become more complicated polynomials. Formula for negative moments can also be obtained from (33-36) performing the same limit.

\subsubsection{Random Statistical Mechanics Model Associated to Laguerre Ensemble}

In the corresponding disordered model, LCRG with a background confining potential (154) we find

$$
\begin{aligned}
& \overline{<z>_{\beta}}=\frac{1}{\beta}+\bar{a}+\beta \\
& \overline{<z^{2}>_{\beta}}-\overline{<z>^{2}}=\left(\frac{1}{\beta}+\beta\right)\left(\frac{1}{\beta}+\beta+\bar{a}\right)
\end{aligned}
$$

\footnotetext{
10 Note that positive moments for the Laguerre ensemble were also presented in [31] in an equivalent, but less explicit form.
} 
Freezing of these manifestly duality invariant expressions lead to

$$
\overline{z_{m}}=2+\bar{a}, \quad \overline{z_{m}^{2}}-{\overline{z_{m}}}^{2}=2(2+\bar{a})
$$

In the absence of disorder the absolute minimum is at $z_{m}^{0}=\bar{a}$, hence the random potential now tends to push the minimum towards the larger positive $z$ (i.e. to unbind the particle). One finds a few higher moments

$$
\overline{z_{m}^{3}}=(2+a)(23+a(10+a)), \quad \overline{z_{m}^{4}}=(2+a)(168+a(99+a(18+a)))
$$

whose associated cumulants have simpler expressions

$$
\begin{aligned}
& {\overline{z_{m}^{3}}}^{c}=7(2+\bar{a}), \quad{\overline{z_{m}^{4}}}^{c}=(\bar{a}-32)(2+\bar{a}) \\
& {\overline{z_{m}^{5}}}^{c}=4(2+\bar{a})(42-5 \bar{a}), \quad{\overline{z_{m}^{6}}}^{c}=2(2+\bar{a})(458+\bar{a}(-147+2 \bar{a}))
\end{aligned}
$$

\subsection{Gaussian-Hermite Ensemble}

Let us now turn to the Gaussian ensemble. This model is of great interest as its disordered statistical mechanics is associated to the solution of the one-dimensional decaying Burgers equation with a random initial condition and of viscosity $\sim 1 / \beta$. The velocity is the gradient of a potential, and the initial condition is chosen to correspond to a log-correlated random potential. The one-space point statistics of the velocity at any later time is then exactly associated to the statistical model of the Gaussian ensemble, as we showed in [49]. The freezing transition at $\beta=1$ corresponds to a transition in the Burgers dynamics from a Gaussian phase, to a shock-dominated phase. For more details on the correspondence between the two problems, we refer the reader to [49] where the model is introduced and analyzed. We use the same conventions as in that work. Defining the new variable $z$ :

$$
z=\sqrt{8 \bar{a}}\left(y-\frac{1}{2}\right), \quad a=\beta \bar{a}
$$

in terms of the Jacobi variable $y \in[0,1]$, we obtain the moments of the Gaussian ensemble by taking $b=a \rightarrow+\infty$ in the general formula (33-34). This limit is not simple. First, raising (171) to the power $z^{k}$ requires adding contributions of various moments $<y^{p}>$ of degree $p \leq k$. Second, in each such moment there is no obvious term by term simplification in the large $a=b$ limit. As one sees on (33-34), each term $A_{\lambda}$ is superficially of order one, hence multiple cancellations do occur in the sum so that the end result is of order $1 / a^{k / 2}$ at large $a$. The calculation is thus handled using Mathematica, which allows to obtain moments to high degrees.

Setting $n=0$, we then obtain the first non-trivial cumulants of $\overline{p_{\beta}(z)}$. Note that the weight factor is now $e^{-\beta z^{2} / 2}$ hence the disordered model corresponds to a particle in a LCGP in presence of a quadratic confining background potential $V_{0}(z)=z^{2} / 2$ at inverse temperature $\beta$. We obtain

$$
\begin{aligned}
& \overline{<z^{2}>_{\beta}}=\beta+\beta^{-1} \\
& \overline{<z^{4}>_{\beta}}{ }^{c}=\overline{<z^{4}>_{\beta}}-3 \overline{<z^{2}>_{\beta}}=-1
\end{aligned}
$$

and we list here the next three ones i.e. ${\overline{<z^{k}>_{\beta}}}^{c}$ for $k=6,8,10$

$$
\left\{2\left(\beta+\beta^{-1}\right),-2\left(3 \beta^{2}+13+3 \beta^{-2}\right), 12\left(\beta+\beta^{-1}\right)\left(2 \beta^{2}+23+2 \beta^{-2}\right)\right\}
$$


These expressions are identical to the ones calculated in [49] using there a much more painstaking method. They are manifestly duality-invariant and freeze at $\beta=1$, from which one can read the expressions for the corresponding moments of the position of the minimum $z_{m}$ (which we do not write here in detail). Since, as discussed there, the Burgers velocity $v$ in the inviscid limit is equal to the position of the minimum $v \equiv z_{m}$ in the Gaussian ensemble problem, this leads to non-trivial predictions for the moments of the PDF of these two quantities, some of which were numerically checked there.

\section{Discussion and Conclusions}

\subsection{Numerical Verification}

We now compare our predictions for the position $x_{m}$ of the maximum of the GUE-CP with the results of direct numerical simulations of GUE polynomials for matrices of growing size $N$, performed by Nick Simm, who we also thank for the detailed analysis of the data.

In Fig. 1 we show the histogram of the full PDF of the position $x_{m}$. For the sake of comparison we also plotted the semi-circle density of eigenvalues, which as discussed above, has the same first negative moment. The data suggest that, although the distribution of the maximum if clearly not given by the semi-circular law, the edge behaviors of the two distributions are numerically close.

Next, in Figs. 2 and 3 we are plotting the values of the second moment and of the kurtosis as compared to the Prediction 1. While the detailed analysis of finite size effects is left for the future, we plotted the data against the finite size scale $1 /\left[10(\ln N)^{3}\right]$, which we found appropriate.

We see a rather good agreement for the extrapolated values of the second moment, and still reasonable agreement for the kurtosis. In Fig. 4, we also plot the first inverse moment, which shows rather good convergence to the predicted value 2 . The second inverse moment, predicted to diverge, is also shown in Fig. 5.

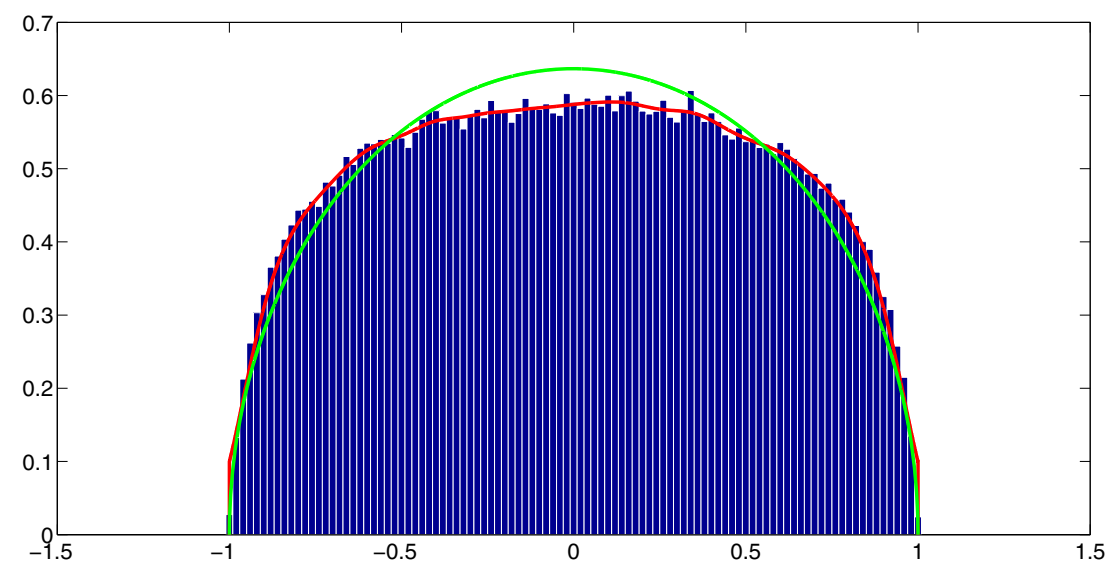

Fig. 1 Histogram of values $x_{m}$ for the position of the maximum of the characteristic polynomial for size $N=3000$ GUE matrices with 250, 000 realizations. We use the numerical method described in Section 3 of [21]. The curve fitting the histogram (red) differs from the semi-circular density (green) at most by 0.099 


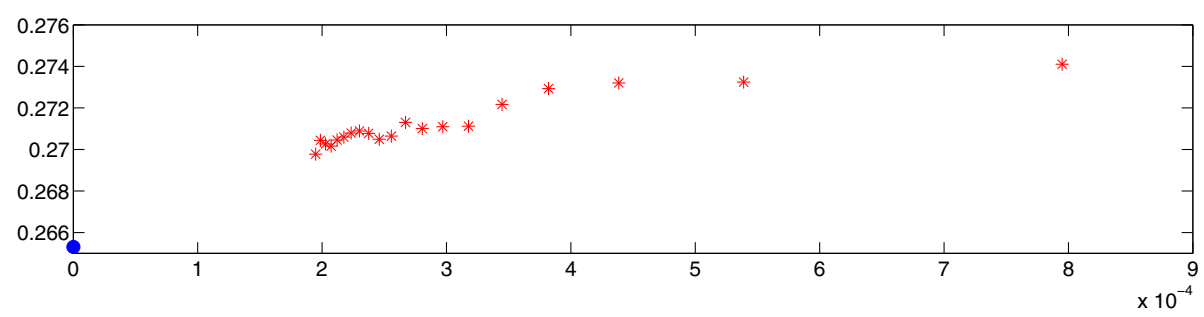

Fig. 2 Variance of the position of the maximum of the characteristic polynomial for 20 equally spaced data points corresponding to size $N=150$ up to size $N=3000$ GUE matrices with 250,000 realizations. The $x$ axis has been chosen as $1 /\left[10(\ln N)^{3}\right]$. The blue point is the prediction (123)

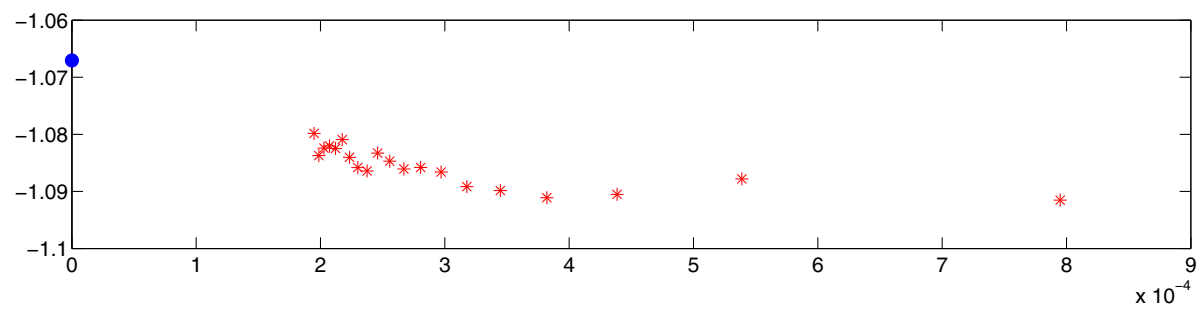

Fig. 3 Kurtosis of the position of the maximum of the characteristic polynomial, same samples and $x$ axis scale as in Fig.2. The blue point is the Prediction 1

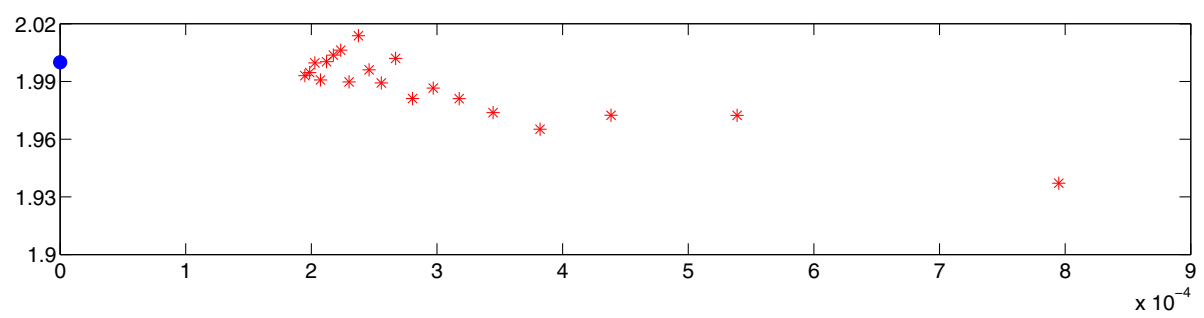

Fig. 4 Inverse moment of the position of the maximum of the characteristic polynomial, same samples and $x$ axis scale as in Fig.2. The blue point is the prediction (136)

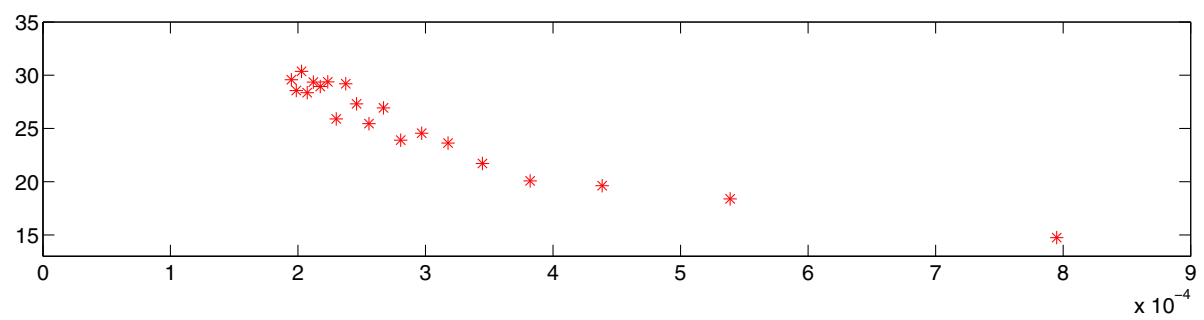

Fig. 5 Inverse moment of the position of the maximum of the characteristic polynomial, same samples and $x$ axis scale as in Fig.2. The prediction is a divergence of the moment as $N \rightarrow+\infty$

In conclusion the agreement with the predictions is reasonable, and for some observables, excellent. We hope that increasing both the number of realizations and the value of the parameter $N$ should lead to further improvement, but such a programme is challenging computationally and is left for future research. 


\subsection{Conclusions}

In this paper we developed a systematic approach to investigating statistical properties of the position $x_{m}$ of the global extremum (maximum or minimum) for appropriately regularized logarithmically-correlated gaussian (LCG) processes in an interval. We explicitly treat three processes of that kind: the logarithm of the Gaussian unitary ensemble (GUE) characteristic polynomial, the log-correlated potential in presence of edge charges, and the Fractional Brownian motion with Hurst index $H \rightarrow 0$, The distribution of $x_{m}$ is characterized through its positive integer moments $\mathbb{E}\left\{\left[x_{m}\right]^{k}\right\}$ for which we provided an explicit, closed form expression in terms of a sum over partitions of the integer $k$. Our approach is based on the idea of interpreting LCGs as a random potential so that the associated Boltzmann-Gibbs measure in the limit of zero temperature $T \rightarrow 0$ concentrates around the coordinates of the global minimum for the potential. Our main technical instrument of analysis is then combining the replica trick representation for the Boltzmann-Gibbs average of $\left[x_{m}\right]^{k}$ with the possibility of exact evaluation of that average by mapping it to the moment problem for $\beta$-Jacobi ensemble of random matrices. To perform the latter we used a method based on Jack polynomials expansion and Macdonald-Kadell integral, with alternative routes possible via Borodin-Gorin moment formula. The latter appproach provides also some expressions for negative integer moments. Our calculations yield explicit formulae for the moments in the high-temperature phase $T>T_{c}$, which can be further continued to $T<T_{c}$ by exploiting the FreezingDuality Conjecture, and in this way provide for $T \rightarrow 0$ the sought for expressions for $\mathbb{E}\left\{\left[x_{m}\right]^{k}\right\}$. Although any integer moment of $x_{m}$ can be with due effort calculated in that way, it remains a challenge to convert such information into the appropriate generating function for the distribution of $x_{m}$. Doing this would allows to understand, e.g., far tails of the latter distribution, and we leave that and other interesting question for the future work. Note that we have also provided results about correlations between position and value of the maximum, and a general method to calculate conditional moments. The determination of the full the joint probability is also left for the future. We also used the numerical data provided by Nick Simm to test our predictions for the moments of the position for the maximum in GUE case. The agreement with our theory is reasonable, and for some observables, excellent.

The application of our method to a non-stationary process, the fBm0, poses several questions. While the analytical continuation to $n=0$ seems to be benign for the moments of $x_{m}$, and leads to the interesting Prediction 3, extension to determine the PDF of the global minimum seems to fail, as discussed in the Appendix 7. It would thus be desirable in near future to find a way around this problem, as well as to check the predictions for the moments against numerical simulations of the process $B_{0}^{(\eta)}(x)$. Unfortunately generating many instances with reliable precision seems to be extremely time-demanding. To this end recall that there exists an intimate relation between $B_{0}^{(\eta)}(x)$ and the behaviour of the (increments of) GUE characteristic polynomials, though at a different, so-called "mesoscopic" spectral scales [1], negligible in comparision with the interval $[-1,1]$. Clearly, such restriction makes the problem challenging and we leave numerical verification/falsification of the Prediction 3 for the future.

We believe that our analysis of $\beta$-Jacobi ensemble is interesting in its own right and complements one that has appeared in the very recent work by Mezzadri and Reynolds [31]. We have also presented conjectures for moments in related ensemble.

Acknowledgments The authors are very grateful to Nick Simm for kindly providing numerical data on argmax of GUE, as well as to Alexei Borodin and Vadim Gorin for bringing their methods to our attention, for writing the Appendix 1 in the present paper, and for indicating relevant references to us. We would like to warmly thank the anonymous referee for suggesting to use formulas $(80,81)$ for obtaining an explicit expression 
for the negative moments, Alberto Rosso for a lively discussions at the early stage of the project, and Dima Savin for guiding us to the literature on the moments of Jacobi density. We also thank F. Mezzadri and A. Reynolds for informing us on their moment formulae prior to publication. Kind hospitality of the Newton Institute, Cambridge during the program "Random Geometry" as well as of the Simon Center for Geometry and Physics in Stony Brook, where this research was completed, is acknowledged with thanks. YF was supported by EPSRC Grants EP/J002763/1 "Insights into Disordered Landscapes via Random Matrix Theory and Statistical Mechanics" and EP/N009436/1 "The many faces of random characteristic polynomials". PLD was supported by PSL Grant ANR-10-IDEX-0001- 02-PSL.

Open Access This article is distributed under the terms of the Creative Commons Attribution 4.0 International License (http://creativecommons.org/licenses/by/4.0/), which permits unrestricted use, distribution, and reproduction in any medium, provided you give appropriate credit to the original author(s) and the source, provide a link to the Creative Commons license, and indicate if changes were made.

\section{Appendix 1: Contour Integral Formulas for Jacobi Ensemble}

Consider the $N$-particle Jacobi ensemble, which is a probability distribution on $N$-tuples of reals $0 \leq \mathbf{r}_{1} \leq \mathbf{r}_{2} \leq \cdots \leq \mathbf{r}_{N} \leq 1$ with density

$$
\mathbb{P}^{\alpha, M, \theta}(\mathbf{r} \in[h, h+d h])=\mathrm{const} . \prod_{1 \leq i<j \leq N}\left(h_{j}-h_{i}\right)^{2 \theta} \prod_{i=1}^{N} h_{i}^{\theta \alpha-1}\left(1-h_{i}\right)^{\theta(M-N+1)-1} d h_{i},
$$

where $M \geq N$ is an integer, and $\alpha>0, \theta>0$ are two real parameters. $2 \theta$ is customary called $\beta$ in the random matrix theory.

Theorem 1 For $k=1,2, \ldots$ the expectation of $\sum_{i=1}^{N}\left(\mathbf{r}_{i}\right)^{k}$ with respect to the measure (175) is given by

$$
\begin{aligned}
& \frac{(-\theta)^{-1}}{(2 \pi \mathbf{i})^{k}} \oint \ldots \oint \frac{1}{\left(u_{2}-u_{1}+1-\theta\right) \cdots\left(u_{k}-u_{k-1}+1-\theta\right)} \\
& \quad \times \prod_{i<j} \frac{\left(u_{j}-u_{i}\right)\left(u_{j}-u_{i}+1-\theta\right)}{\left(u_{j}-u_{i}-\theta\right)\left(u_{j}-u_{i}+1\right)}\left(\prod_{i=1}^{k} \frac{u_{i}-\theta}{u_{i}+(N-1) \theta} \cdot \frac{u_{i}-\theta \alpha}{u_{i}-\theta \alpha-\theta M} d u_{i}\right),
\end{aligned}
$$

where all the contours enclose singularities at $(1-N) \theta$ and not at $\theta \alpha+\theta M,\left|u_{1}\right| \ll\left|u_{2}\right| \ll$ $\cdots \ll\left|u_{k}\right|$.

Theorem 2 For any positive integer $k<\theta \alpha$ the expectation of $\sum_{i=1}^{N}\left(\mathbf{r}_{i}\right)^{-k}$ with respect to the measure (175) is given by

$$
\begin{aligned}
& \frac{\theta^{-1}}{(2 \pi \mathbf{i})^{k}} \oint \ldots \oint \frac{1}{\left(u_{2}-u_{1}-1+\theta\right) \cdots\left(u_{k}-u_{k-1}-1+\theta\right)} \\
& \quad \times \prod_{i<j} \frac{\left(u_{j}-u_{i}\right)\left(u_{j}-u_{i}-1+\theta\right)}{\left(u_{j}-u_{i}+\theta\right)\left(u_{j}-u_{i}-1\right)}\left(\prod_{i=1}^{k} \frac{u_{i}+N \theta}{u_{i}} \cdot \frac{u_{i}+1-\theta \alpha-M \theta}{u_{i}+1-\theta \alpha} d u_{i}\right),
\end{aligned}
$$

where all the contours enclose singularities at 0 and not at $\theta \alpha-1,\left|u_{1}\right| \ll\left|u_{2}\right| \ll \cdots \ll\left|u_{k}\right|$.

Remark 3 There exist similar contour integral formulas for the expectations of the powers $\left(\sum_{i=1}^{N}\left(\mathbf{r}_{i}\right)^{k}\right)^{m}$ with $m=1,2, \ldots$ and $k$ being both positive and negative integers. They are obtained by iterating the results of Propositions 6, 7 below. 
In the rest of this section we prove Theorems 1 and 2.

The starting point of our proof is a formula from [58] which we now present. Let $\Lambda$ be the algebra of symmetric polynomials in infinitely many variables $x_{1}, x_{2}, \ldots$ This algebra is naturally identified with polynomial algebra $\mathbb{C}\left[p_{1}, p_{2}, \ldots\right]$, where $p_{k}$ are Newton power sums:

$$
p_{k}=x_{1}^{k}+x_{2}^{k}+x_{3}^{k}+\ldots, \quad k=1,2, \ldots
$$

We also need a distinguished linear basis in $\Lambda$ consisting of Macdonald polynomials $P_{\lambda}(\cdot ; q, t)$, which depend on two parameters $q$ and $t$; here and below we use the notations of [54] and $\lambda=\lambda_{1} \geq \lambda_{2} \geq \cdots \geq 0$ is a Young diagram.

Proposition 4 Define a differential operator $\mathfrak{D}_{n}$ acting in $\Lambda$ via

$$
\begin{aligned}
\mathfrak{D}_{n}= & \frac{(-1)^{n-1}}{(2 \pi \mathbf{i})^{n}} \oint \ldots \oint \frac{\sum_{i=1}^{n} \frac{z_{n}}{z_{i}(t / q)^{n-i}}}{\left(1-\frac{q z_{2}}{t z_{1}}\right) \cdots\left(1-\frac{q z_{n}}{t z_{n-1}}\right)} \prod_{i<j} \frac{\left(1-\frac{z_{i}}{z_{j}}\right)\left(1-\frac{t z_{i}}{q z_{j}}\right)}{\left(1-t \frac{z_{i}}{z_{j}}\right)\left(1-q^{-1} \frac{z_{i}}{z_{j}}\right)} \\
& \times \exp \left(\sum_{k=1}^{\infty} q^{-k}\left(1-t^{k}\right) \frac{z_{1}^{-k}+\cdots+z_{n}^{-k}}{k} p_{k}\right) \exp \left(\sum_{k=1}^{\infty} \frac{z_{1}^{k}+\cdots+z_{n}^{k}}{k}\left(1-q^{k}\right) \frac{\partial}{\partial p_{k}}\right) \\
& \times \frac{d z_{1}}{z_{1}} \cdots \frac{d z_{n}}{z_{n}},
\end{aligned}
$$

where the contours are circles around 0 satisfying $\left|z_{1}\right| \ll\left|z_{2}\right| \ll \cdots \ll\left|z_{n}\right|$, and $p_{k}$ means the operator of multiplication by $p_{k}$. Then Macdonald polynomials are eigenfunctions of $\mathfrak{D}_{n}$, i.e.

$$
\mathfrak{D}_{n} P_{\lambda}(\cdot ; q, t)=\left(1-t^{n}\right) \sum_{i=1}^{\infty}\left(q^{-\lambda_{i}} t^{i-1}\right)^{n} P_{\lambda}(\cdot ; q, t) .
$$

Proof In slightly different notations this is [58, Theorem 1.2].

Next, we set $x_{N+1}, x_{N+2}, \ldots$ equal to 0 and apply the above operators to an $N$-variable product function $f\left(x_{1}\right) \cdots f\left(x_{N}\right)$ (which belongs to the space of symmetric power series in $\left.x_{1}, \ldots, x_{N}\right)$. Then we get

$$
\begin{aligned}
\mathfrak{D}_{n} & \prod_{i=1}^{N} f\left(x_{i}\right)=\left(\prod_{i=1}^{N} f\left(x_{i}\right)\right) \frac{(-1)^{n-1}}{(2 \pi \mathbf{i})^{n}} \oint \ldots \oint \frac{\sum_{i=1}^{n} \frac{z_{n}}{z_{i}(t / q)^{n-i}}}{\left(1-\frac{q z_{2}}{t z_{1}}\right) \cdots\left(1-\frac{q z_{n}}{t z_{n-1}}\right)} \\
& \times \prod_{i<j} \frac{\left(1-\frac{z_{i}}{z_{j}}\right)\left(1-\frac{t z_{i}}{q z_{j}}\right)}{\left(1-t \frac{z_{i}}{z_{j}}\right)\left(1-q^{-1} \frac{z_{i}}{z_{j}}\right)}\left(\prod_{i=1}^{n} \prod_{a=1}^{N} \frac{z_{i}-t q^{-1} x_{a}}{z_{i}-q^{-1} x_{a}}\right) \prod_{i=1}^{n} \frac{f\left(z_{i}\right)}{f\left(q z_{i}\right)} \frac{d z_{i}}{z_{i}} .
\end{aligned}
$$

In the last formula the contours are large circles (this is because the integrand needs to be decomposable into a symmetric power series in the variables $x_{i}$ to justify the computation).

At this point we can pass from formal point of view based on the algebra $\Lambda$ to the analytic one and view $q, t$ and $x_{i}$ as real (or complex) numbers. Our next step is the following limit transition:

$$
\varepsilon \rightarrow 0, \quad q=\exp (-\varepsilon), \quad t=q^{\theta}, \quad z_{i}=\exp \left(\varepsilon u_{i}\right), \quad x_{i}=\exp \left(\varepsilon y_{i}\right), \quad \lambda_{i}=\varepsilon^{-1} r_{i} .
$$


The Macdonald polynomials $P_{\lambda}$ are shown in [59] to converge to the Heckman-Opdam hypergeometric functions $H O_{r}\left(y_{1}, \ldots, y_{N} ; \theta\right)$ in this limit regime:

$$
\lim _{\varepsilon \rightarrow 0} P_{\lambda}\left(x_{1}, \ldots, x_{N} ; q, t\right)=H O_{r}\left(y_{1}, \ldots, y_{N} ; \theta\right) .
$$

In order to pass to the limit $\varepsilon \rightarrow 0$ in the operators $\mathfrak{D}_{n}$ we need to deform the integration contours so that they enclose the singularities in $q^{-1} x_{i}$ but not 0 . Due to the ordering of the contours we should do this one after another: first deform the $z_{1}$ contour, then deform the $z_{2}$ contour, etc. In principle, in this process we get $2^{n}$ terms obtained by taking the residues at 0 in a subset of variables $z_{1}, \ldots, z_{n}$.

Lemma 5 Only subsets of the form $z_{k}, z_{k+1}, \ldots, z_{\ell}, 0 \leq k<\leq l \leq n$ give non-zero residues in (179).

Proof Recall that we should compute the residues sequentially, from $z_{1}$ to $z_{n}$. Suppose that we started from $z_{k}$. Then the residue is $n-1$ dimensional integral, in which the factors in the second line of (179) are still the same (with additional prefactor $t^{N}$ ), while the factor in the first line transforms into

$$
(-1) \frac{\frac{z_{n}}{z_{k+1}(t / q)^{n-k-1}}}{\left(1-\frac{q z_{2}}{t z_{1}}\right) \cdots\left(1-\frac{q z_{k-1}}{t z_{k-2}}\right)\left(1-\frac{q z_{k+2}}{t z_{k+1}}\right) \cdots\left(1-\frac{q z_{n}}{t z_{n-1}}\right)}
$$

Note that if at the next step we do not take the residue in $z_{k+1}$, then all further residues in $z_{i}$, $i>k+1$ will be zero - the integrand will simply have no poles at 0 in these variables. If we take the residue in $z_{k+1}$, then we get

$$
(-1)^{2} \frac{\frac{z_{n}}{z_{k+2}(t / q)^{n-k-2}}}{\left(1-\frac{q z_{2}}{t z_{1}}\right) \cdots\left(1-\frac{q z_{k-1}}{t z_{k-2}}\right)\left(1-\frac{q z_{k+3}}{t z_{k+2}}\right) \cdots\left(1-\frac{q z_{n}}{t z_{n-1}}\right)}
$$

which still has the same form and, thus, we can continue in the same way.

In particular, if we take the residue at 0 with respect to all variables $z_{1}, \ldots, z_{n}$, then we get

$$
(-1)^{n-1} t^{N n}
$$

Note that $(-1)^{n-1}$ cancels out with the integral prefactor. Let us pass to the operator

$$
\mathfrak{D}_{n}-t^{N n}
$$

On one hand, this operator is given by the expansion into $n(n+1) / 2$ integrals of various dimensions integrated around $q^{-1} x_{i}$ but not 0 . On the other hand, its eigenvalues on Macdonald polynomials in $N$ variables are

$$
\left(1-t^{n}\right)\left(\sum_{i=1}^{N}\left(q^{-\lambda_{i}} t^{i-1}\right)^{n}+\sum_{i=N+1}^{N}\left(t^{i-1}\right)^{n}\right)-t^{N n}=\left(1-t^{n}\right) \sum_{i=1}^{N}\left(q^{-\lambda_{i}} t^{i-1}\right)^{n}
$$

The eigenvalues of $\varepsilon^{-1}\left(\mathfrak{D}_{n}-t^{N n}\right)$ converge in the limit regime (180) to

$$
\theta n \sum_{i=1}^{N} \exp \left(n r_{i}\right)
$$

Thus, taking into account (181), we conclude that integral representation for $\varepsilon^{-1}\left(\mathfrak{D}_{n}-t^{N n}\right)$ should also converge. The $n$-dimensional integral here converges to 


$$
\begin{aligned}
& \left(\prod_{i=1}^{N} f\left(y_{i}\right)\right) \frac{(-1)^{n-1}}{(2 \pi \mathbf{i})^{n}} \oint \oint \frac{n}{\left(u_{1}-u_{2}+1-\theta\right) \cdots\left(u_{n-1}-u_{n}+1-\theta\right)} \\
& \quad \times \prod_{i<j} \frac{\left(u_{j}-u_{i}\right)\left(u_{j}-u_{i}-1+\theta\right)}{\left(u_{j}-u_{i}+\theta\right)\left(u_{j}-u_{i}-1\right)}\left(\prod_{i=1}^{n} \prod_{a=1}^{N} \frac{u_{i}-y_{a}+\theta-1}{u_{i}-y_{a}-1}\right) \prod_{i=1}^{n} \frac{f\left(u_{i}\right)}{f\left(u_{i}-1\right)} d u_{i},
\end{aligned}
$$

where all the contours enclose singularities at $y_{a}+1$ and $\left|u_{1}\right| \ll\left|u_{2}\right| \ll \cdots \ll\left|u_{n}\right|$.

Note that is was important to have $n-1$ factors in the first line. Indeed, each such factor produced $\varepsilon^{-1}$. On the other hand, $\varepsilon^{n}$ was produced by the change of variables. Together with additional $\varepsilon^{-1}$ in the definition of our limit transition this gave precisely the constant order as $\varepsilon \rightarrow 0$. Now note that when we computed the residues as in Lemma 5 , then when $1<k<n$, the $m$-dimensional integral would come with less than $m-1$ factors in the first line. Indeed, this is clearly visible in (182), (183). If follows that such terms vanish in our limit transition. Therefore, only terms with $k=1$ or $k=n$ survive. A general term is obtained either by taking the residue in variables

$$
z_{1}, \ldots, z_{\ell}, \quad \ell=1, \ldots, n-1
$$

as in Lemma 5 and then sending $\varepsilon \rightarrow 0$. As a result, we get $n-\ell$ dimensional integral

$$
\begin{aligned}
& \left(\prod_{i=1}^{N} f\left(y_{i}\right)\right) \frac{(-1)^{n-1-\ell}}{(2 \pi \mathbf{i})^{n-\ell}} \oint \oint \frac{1}{\left(u_{\ell+1}-u_{\ell+2}+1-\theta\right) \cdots\left(u_{n-1}-u_{n}+1-\theta\right)} \\
& \quad \times \prod_{i<j} \frac{\left(u_{j}-u_{i}\right)\left(u_{j}-u_{i}-1+\theta\right)}{\left(u_{j}-u_{i}+\theta\right)\left(u_{j}-u_{i}-1\right)}\left(\prod_{i=\ell+1}^{n} \prod_{a=1}^{N} \frac{u_{i}-y_{a}+\theta-1}{u_{i}-y_{a}-1}\right) \prod_{i=\ell+1}^{n} \frac{f\left(u_{i}\right)}{f\left(u_{i}-1\right)} d u_{i},
\end{aligned}
$$

Or we can take the residue in variables

$$
z_{k}, \ldots, z_{n}, \quad, n=1, \ldots, n-1
$$

as in Lemma 5 and then send $\varepsilon \rightarrow 0$. Note that under the identification $k=n+1-\ell$, the integrals have the same integrand. However, the signs appearing when we take residues are different. Namely, when we take residues in $z_{1}, \ldots, z_{\ell}$ we get the sign $(-1)^{\ell}$. On the other hand, when we take residues in $z_{n+1-\ell}, \ldots, z_{n}$ the sign is $(-1)^{\ell-1}$, since we do not get $(-1)$ factor at the very last step. As a conclusion, two such terms precisely cancel out.

Shifting the variables $u \mapsto u+1$ and dividing by $\theta n$ we write the final formula.

Proposition 6 The action of the operator $\mathcal{P}_{n}:=\frac{1}{\theta n} \lim _{\varepsilon \rightarrow 0} \varepsilon^{-1}\left(\mathfrak{D}_{n}-t^{N n}\right)$ with eigenvalues

$$
\mathcal{P}_{n} H O_{r}\left(y_{1}, \ldots, y_{N} ; \theta\right)=\sum_{i=1}^{N} \exp \left(n r_{i}\right) H O_{r}\left(y_{1}, \ldots, y_{N} ; \theta\right),
$$

on a function $f\left(y_{1}\right) \cdots f\left(y_{N}\right)$ can be computed via 


$$
\begin{aligned}
\frac{\mathcal{P}_{n} \prod_{i=1}^{N} f\left(y_{i}\right)}{\prod_{i=1}^{N} f\left(y_{i}\right)}= & \frac{\theta^{-1}}{(2 \pi \mathbf{i})^{n}} \oint \ldots \oint \frac{1}{\left(u_{2}-u_{1}-1+\theta\right) \cdots\left(u_{n}-u_{n-1}-1+\theta\right)} \\
& \times \prod_{i<j} \frac{\left(u_{j}-u_{i}\right)\left(u_{j}-u_{i}-1+\theta\right)}{\left(u_{j}-u_{i}+\theta\right)\left(u_{j}-u_{i}-1\right)}\left(\prod_{i=1}^{n} \prod_{a=1}^{N} \frac{u_{i}-y_{a}+\theta}{u_{i}-y_{a}}\right) \\
& \times \prod_{i=1}^{n} \frac{f\left(u_{i}+1\right)}{f\left(u_{i}\right)} d u_{i}
\end{aligned}
$$

where all the contours enclose singularities at $y_{a}, f(u+1) / f(u)$ is analytic inside the contours and $\left|u_{1}\right| \ll\left|u_{2}\right| \ll \cdots \ll\left|u_{n}\right|$.

Further, note that in the operator $\mathfrak{D}_{n}-t^{N n}$ we can freely invert the variables $(q, t) \mapsto$ $\left(q^{-1}, t^{-1}\right)$. Since, the Macdonald polynomials are invariant under this change (cf. [54]), they are still eigenfunctions. Moreover, since we never used the fact $0<t, q<1$ in the proofs, all the integral representations are still valid. Thus, we arrive at:

Proposition 7 The action of the operator $\widehat{\mathcal{P}}_{n}$ with eigenvalues

$$
\widehat{\mathcal{P}}_{n} H O_{r}\left(y_{1}, \ldots, y_{N} ; \theta\right)=\sum_{i=1}^{N} \exp \left(-n r_{i}\right) H O_{r}\left(y_{1}, \ldots, y_{N} ; \theta\right),
$$

on a function $f\left(y_{1}\right) \cdots f\left(y_{N}\right)$ can be computed via

$$
\begin{aligned}
\frac{\mathcal{P}_{n} \prod_{i=1}^{N} f\left(y_{i}\right)}{\prod_{i=1}^{N} f\left(y_{i}\right)}= & \frac{(-\theta)^{-1}}{(2 \pi \mathbf{i})^{n}} \oint \ldots \oint \frac{1}{\left(u_{2}-u_{1}+1-\theta\right) \cdots\left(u_{n}-u_{n-1}+1-\theta\right)} \\
& \times \prod_{i<j} \frac{\left(u_{j}-u_{i}\right)\left(u_{j}-u_{i}+1-\theta\right)}{\left(u_{j}-u_{i}-\theta\right)\left(u_{j}-u_{i}+1\right)}\left(\prod_{i=1}^{n} \prod_{a=1}^{N} \frac{u_{i}-y_{a}-\theta}{u_{i}-y_{a}}\right) \\
& \times \prod_{i=1}^{n} \frac{f\left(u_{i}-1\right)}{f\left(u_{i}\right)} d u_{i}
\end{aligned}
$$

where all the contours enclose singularities at $y_{a}, f(u-1) / f(u)$ is analytic inside the contours and $\left|u_{1}\right| \ll\left|u_{2}\right| \ll \cdots \ll\left|u_{n}\right|$.

Now we are ready to prove the contour integral formulas for the Jacobi ensemble.

Proof of Theorem 2 This is essentially a corollary of Proposition 6 and the results of [59] and below we sketch the proof omitting some technical details, cf. [59, Section 2.3] for similar arguments.

The Cauchy identity for the Macdonald polynomials yields for $M \geq N$

$$
\sum_{\lambda=\left(\lambda_{1} \geq \lambda_{2} \geq \cdots \geq \lambda_{N} \geq 0\right)} \frac{P_{\lambda}\left(x_{1}, \ldots, x_{N} ; q, t\right) P_{\lambda}\left(\hat{x}_{1}, \ldots, \hat{x}_{M} ; q, t\right)}{\left\langle P_{\lambda}, P_{\lambda}\right\rangle}=\prod_{i=1}^{N} \prod_{j=1}^{M} \frac{\left(t x_{i} \hat{x}_{j} ; q\right)_{\infty}}{\left(x_{i} \hat{x}_{j} ; q\right)_{\infty}},
$$

where $(a ; q)_{\infty}$ is the $q$-Pochhammer symbol, $(a ; q)_{\infty}=\prod_{\ell=0}^{\infty}\left(1-a q^{\ell}\right)$, and $\left\langle P_{\lambda}, P_{\lambda}\right\rangle$ are certain explicit constants, which can be found e.g. in [54, Chapter VI]. We further do the following three steps:

(i) Apply $\varepsilon^{-1}\left(\mathfrak{D}_{k}-t^{N k}\right)$ to both sides of (187) and then divide them by $\prod_{i=1}^{N} \prod_{j=1}^{M} \frac{\left(t x_{i} \hat{x}_{j} ; q\right)_{\infty}}{\left(x_{i} \hat{x}_{j} ; q\right)_{\infty}}$. 
(ii) Set $x_{i}=t^{i-1}$ and $\hat{x}_{j}=t^{\alpha} t^{j-1}$ and send $\varepsilon \rightarrow 0$ in the limit regime (180).

(iii) Evaluate the $\varepsilon \rightarrow 0$ limit of both sides using Proposition 6 .

We claim that the resulting identity is precisely the statement of Theorem 2. Indeed, it is shown in [59, Theorem 2.8] that as $\varepsilon \rightarrow 0$,

$$
\left(\prod_{i=1}^{N} \prod_{j=1}^{M} \frac{\left(t x_{i} \hat{x}_{j} ; q\right)_{\infty}}{\left(x_{i} \hat{x}_{j} ; q\right)_{\infty}}\right)^{-1} \frac{P_{\lambda}\left(x_{1}, \ldots, x_{N} ; q, t\right) P_{\lambda}\left(\hat{x}_{1}, \ldots, \hat{x}_{M} ; q, t\right)}{\left\langle P_{\lambda}, P_{\lambda}\right\rangle}
$$

converges to the density of the Jacobi ensemble (175) in variables $\mathbf{r}_{i}=\exp \left(-r_{i}\right)$. Together with the eigenrelation for $\mathcal{P}_{n}$ of Proposition 6 this implies that the left-hand side of the identity is the expectation of $\sum_{i=1}^{N}\left(\mathbf{r}_{i}\right)^{-k}$. For the right-hand side we use the convergence of the $q$-Pochhammer symbols to the Gamma function as $q \rightarrow 1$ to get the expression of the form (186) with

$$
f(y)=\frac{\Gamma(-y+\theta \alpha)}{\Gamma(-y+\theta \alpha+\theta M)},
$$

which is precisely (177).

Theorem 1 is proven in the same way as Theorem 2, but using Proposition 7 instead of Proposition 6.

\section{Appendix 2: Calculation of Contour Integrals and More Results for Moments}

In this Appendix we give some details of the contour integral calculations, as well as some additional explicit results. All formula presented here have also been obtained our expressions of moments in terms of partitions.

\section{Second Moment}

Consider (102) for $k=2$. One first perform the contour integral over $u_{1}$ on a small circle around 0 . The poles in $u_{1}$ are at $u_{1}=0,2+a+b+2 \beta^{2}, 1+u_{2}, \beta^{2}+u_{2}$. Because of condition $C_{1}$ the last two poles are not encountered. Because of $C_{2}$ neither is the second pole. Hence only the pole in $u_{1}=0$ is picked up and from its residue one gets the remaining integral:

$$
<y^{2}>_{\beta, a, b, n=0}=\frac{\left(a+\beta^{2}+1\right)}{\left(a+2 \beta^{2}+b+2\right)} \int \frac{d u_{2}}{2 i \pi} \frac{u_{2}\left(u_{2}-\left(a+\beta^{2}+1\right)\right)}{\left(u_{2}+1\right)\left(u_{2}+\beta^{2}\right)\left(u_{2}-\left(a+2 \beta^{2}+2\right)\right)}
$$

From the prescription $C_{2}$ only the poles at $u_{2}=-1$ and $u_{2}=-\beta^{2}$ contribute and, summing their residues one obtains (113) in the text.

For completeness we now give the complete $n$ dependence of several results in the text.

For the LCP with edge charges in the case $b=a$ one finds:

$$
<y^{2}>_{\beta, a, a, n}=\frac{2 a^{2}+a\left(\beta^{2}(6-5 n)+6\right)+\beta^{4}(n-1)(3 n-4)+\beta^{2}(9-7 n)+4}{2\left(2 a+\beta^{2}(3-2 n)+2\right)\left(2 a-2 \beta^{2}(n-1)+3\right)}
$$


For the $\mathrm{fBm}$, with $a=2 n \beta^{2}, b=0$ one has

$$
<y^{2}>_{\beta, n}=\frac{\left(\beta^{2}(n+1)+1\right)\left(\beta^{4}\left(n^{2}+n+4\right)+\beta^{2}(n+9)+4\right)}{2\left(6 \beta^{6}+19 \beta^{4}+19 \beta^{2}+6\right)}
$$

For the GUE-CP, setting $a=b=\frac{1+\beta^{2}}{2}$, one finds:

$$
<y^{2}>_{\beta, n}=\frac{\beta^{4}(2 n-3)(3 n-5)+\beta^{2}(32-19 n)+15}{4\left(2 \beta^{2}(n-2)-3\right)\left(\beta^{2}(2 n-3)-4\right)}
$$

\section{Third Moment}

Consider (102) for $k=3$. We take successively the residue at $u_{1}=0$, then at $u_{2}=-1,-\beta^{2}$, which produces two terms, then at $u_{3}=-2,-\beta^{2}$ for the first term, and at $u_{3}=-1,-2 \beta^{2}$ for the second term. The final result for arbitrary $a, b, \beta$ is too heavy to reproduce here. We give here:

- the third cumulant for arbitrary $a, b$, which is slightly simpler:

$$
\overline{\left(y_{m}-\left\langle y_{m}\right\rangle\right)^{3}}=-\frac{(a+2)(b+2)(a-b)\left(7 a^{2}+2 a(7 b+34)+b(7 b+68)+164\right)}{(a+b+4)^{3}(a+b+5)^{2}(a+b+6)^{2}}
$$

and of course vanishes in the case $a=b$. The associated skewness is

$$
S k=\frac{(b-a)(a+b+5)\left(7 a^{2}+2 a(7 b+34)+b(7 b+68)+164\right)}{\sqrt{a+2} \sqrt{b+2}(a+b+6)^{2}(2 a+2 b+9)^{3 / 2}}
$$

to be compared with the skewness for the measure $y^{a}(1-y)^{b}$, which is

$$
S k_{0}=-\frac{2(a-b) \sqrt{a+b+3}}{\sqrt{a+1} \sqrt{b+1}(a+b+4)}
$$

- we have checked that the result for the third moment for $a=b$, and arbitrary $\beta, n$

$$
<\left(y-\frac{1}{2}\right)^{3}>_{\beta, a, a, n}=0
$$

is consistent with the symmetry $y \rightarrow 1-y$.

\section{Fourth Moment}

Consider now (102) for $k=4$. The sequence of poles is the same as for $k=3$ for $u_{1}, u_{2}, u_{3}$, except for the $u_{4}$ integration which contains now four terms and picks poles at $u_{4}=-3,-\beta^{2}$; $u_{4}=-2,-2 \beta^{2},-1-\beta^{2}$ (twice) and $u_{4}=-1,-3 \beta^{2}$. Clearly this is a simple regular structure which carries on to higher moments. While the pole structure is simple, we were not able to find a systematics for the residue valid to any order.

Again, the final result for arbitrary $a, b, \beta$ is too heavy to reproduce here. We give:

- the fourth moment, cumulant and kurtosis for the GUE-CP associated statistical model with $b=a=\frac{1+\beta^{2}}{2}$, at arbitrary $\beta \leq 1$ :

$$
\overline{<y^{4}>_{\beta}}=\frac{252 \beta^{8}+1195 \beta^{6}+1918 \beta^{4}+1195 \beta^{2}+252}{64\left(\beta^{2}+2\right)\left(2 \beta^{2}+1\right)\left(3 \beta^{2}+4\right)\left(4 \beta^{2}+3\right)}
$$




$$
\begin{aligned}
& \overline{<x^{4}>_{\beta}}=\frac{12 \beta^{8}+59 \beta^{6}+98 \beta^{4}+59 \beta^{2}+12}{4\left(\beta^{2}+2\right)\left(2 \beta^{2}+1\right)\left(3 \beta^{2}+4\right)\left(4 \beta^{2}+3\right)} \\
& \overline{<x^{4}>_{\beta}}-3{\overline{<x^{2}>_{\beta}}}^{2} \\
& =-\frac{72 \beta^{12}+540 \beta^{10}+1549 \beta^{8}+2170 \beta^{6}+1549 \beta^{4}+540 \beta^{2}+72}{4\left(\beta^{2}+2\right)\left(2 \beta^{2}+1\right)\left(3 \beta^{2}+4\right)^{2}\left(4 \beta^{2}+3\right)^{2}} \\
& \mathrm{Ku}=-\frac{72 \beta^{12}+540 \beta^{10}+1549 \beta^{8}+2170 \beta^{6}+1549 \beta^{4}+540 \beta^{2}+72}{4\left(\beta^{2}+2\right)\left(2 \beta^{2}+1\right)\left(3 \beta^{4}+7 \beta^{2}+3\right)^{2}}
\end{aligned}
$$

Setting $\beta=1$, this leads to the predictions given in the text for the maximum of the GUE-CP.

- the fourth moment and cumulants for the statistical model associated to the $\mathrm{fBm} b=$ $a=0$ at arbitrary $\beta \leq 1$ :

$$
\overline{<y^{4}>_{\beta}}=\frac{72 \beta^{8}+382 \beta^{6}+647 \beta^{4}+382 \beta^{2}+72}{6\left(2 \beta^{2}+3\right)\left(2 \beta^{2}+5\right)\left(3 \beta^{2}+2\right)\left(5 \beta^{2}+2\right)}
$$

and the fourth cumulant:

$$
\begin{aligned}
& \overline{\left\langle\left(y-\frac{1}{2}\right)^{4}\right\rangle_{\beta}}-3{\overline{\left\langle\left(y-\frac{1}{2}\right)^{2}\right\rangle_{\beta}}}^{2} \\
& =-\frac{72 \beta^{12}+588 \beta^{10}+1802 \beta^{8}+2599 \beta^{6}+1802 \beta^{4}+588 \beta^{2}+72}{24\left(2 \beta^{2}+3\right)^{2}\left(2 \beta^{2}+5\right)\left(3 \beta^{2}+2\right)^{2}\left(5 \beta^{2}+2\right)}
\end{aligned}
$$

Setting $\beta=1$, this leads to the predictions for the minimum of the fBm given in the text.

\section{Second Negative Moment}

In Eq. (89) for $k=2$, the $u_{1}$ integration gives again the residue at $u_{1}=0$, then the $u_{2}$ integration picks two poles at $u_{2}=1$ and $u_{2}=\beta^{2}$. Since one must avoids the pole at $u_{2}=a$ we need the condition $a>\max \left(1, \beta^{2}\right)$ for the existence of the moment, in which case we find:

$$
\begin{aligned}
& <y^{-2}>\beta, a, b, n \\
& =\frac{\left(a+\beta^{2}+b+\beta^{2}(-n)+1\right)\left(a^{2}-\beta^{2} n\left(a+\beta^{2}+b+1\right)+a b+\beta^{2}+\beta^{4} n^{2}\right)}{(a-1) a\left(a-\beta^{2}\right)}
\end{aligned}
$$

leading, for $n=0$, to the expression given in the text. We have checked that the same expression is obtained from the formula (33-36).

\section{Appendix 3: Numerical Values of Higher Moments}

For completeness, we display values of higher moments for two of our examples.

\section{fBm0}

Let us give the list of even moments $\overline{y_{m}^{k}}, k=6,8, \quad 14$ : 
$\left\{\frac{100691}{648270}, \frac{774289013}{6275253600}, \frac{130667513591}{1272621430080}, \frac{3027227918327}{34360778612160}, \frac{13262063040175909}{171651723898374720}\right\}$

and a longer list of numerical values for $\overline{y_{m}^{k}}, k=6,8, \quad 20$ :

$\{0.155323,0.123388,0.102676,0.0881013,0.0772615,0.0688694,0.0621715,0.0566963\}$

Taking the ratio with $1 /(1+k)$, the moments of the uniform distribution, we obtain

$\{1.08726,1.11049,1.12943,1.14532,1.15892,1.17078,1.18126,1.19062\}$

so they decay slightly slower, meaning more weight near the edges.

The cumulants ${\overline{y_{m}^{k}}}^{c}, k=4,6, . .12$ are given here, together with their numerical value:

$$
\begin{aligned}
& \left\{-\frac{7523}{735000}, \frac{4426903}{810337500},-\frac{125514889189}{19610167500000}, \frac{128185912543691}{9885864269531250},\right. \\
& \left.\quad-\frac{57847493772231002501}{1438689827144882812500}\right\} \\
& \{-0.0102354,0.00546304,-0.0064005,0.0129666,-0.0402085\}
\end{aligned}
$$

as well as the ratio to the corresponding cumulants for the uniform distribution

$$
\{1.22824,1.37669,1.53612,1.71159,1.90626\}
$$

showing again a steady growth.

\section{GUE-CP}

Let us give the list of even moments $\overline{y_{m}^{k}}, k=6,8, . .14$ :

$$
\left\{\frac{731327}{6586272}, \frac{61661759}{772972200}, \frac{31888748599}{523765962720}, \frac{10558018750567}{218042523218520}, \frac{2969274186629889449}{74826879182092612575}\right\}
$$

and a longer list of numerical values for $\overline{y_{m}^{k}}, k=6,8, . .20$ :

$$
\begin{aligned}
& \{0.111038,0.0797723,0.0608836,0.0484218,0.0396819, \\
& 0.0332698,0.0283998,0.024598\}
\end{aligned}
$$

Taking the ratio with the moments of the semi-circle distribution $\rho(x)$, we obtain

$$
1.06017,1.07527,1.08599,1.09353,1.09873,1.10219,1.10432,1.10543
$$

which grow but seem to saturate.

The cumulants ${\overline{y_{m}^{k}}}^{c}, k=4,6, . .12$ are given here, together with their numerical value:

$$
\begin{aligned}
\{- & \frac{541}{115248}, \frac{5665234}{3459233547},-\frac{33307238400767}{26190549031046400}, \frac{6658506099368911}{3882094130126852640}, \\
- & \left.\frac{122275968148461510151943659}{34483421254841283626472130560}\right\} \\
& -0.00469422,0.00163771,-0.00127173,0.00171518,-0.00354593
\end{aligned}
$$


as well as the ratio to the corresponding cumulants for the semi-circle distribution

$\{1.20172,1.34162,1.48828,1.64698,1.82096\}$

which reveal some difference between the two distributions.

\section{Appendix 4: Normalization of Jack Polynomials}

Recalling that $\kappa=1 / \alpha$ and $k=\sum_{i=1}^{\ell(\lambda)} \lambda_{i}$ one finds that the function defined in the text in (60) takes the explicit form, in the two (dual) cases $t=1$ and $t=\alpha$ :

$$
c(\lambda, \alpha, 1)=\alpha^{k} \prod_{i=1}^{\ell(\lambda)}(\kappa(\ell(\lambda)-i+1))_{i} \prod_{1 \leq i<j \leq \ell(\lambda)} \frac{(\kappa(j-i))_{\lambda_{i}-\lambda_{j}}}{(\kappa(j-i+1))_{\lambda_{i}-\lambda_{j}}}
$$

and also

$$
c(\lambda, \alpha, \alpha)=\alpha^{k} \prod_{i=1}^{\ell(\lambda)}(\kappa(\ell(\lambda)-i)+1) \lambda_{i} \prod_{1 \leq i<j \leq \ell(\lambda)} \frac{(\kappa(j-i-1)+1) \lambda_{i}-\lambda_{j}}{(\kappa(j-i)+1) \lambda_{i}-\lambda_{j}}
$$

The product of these two factors being equal to the square of the norm of the Jack polynomial $J_{\lambda}^{(\alpha)}$.

\section{Appendix 5: Averages over Jacobi Measure}

Recalling the definition of the Jacobi ensemble average (30) and taking the ratio of (74) to (29), we obtain the average of the $P^{(1 / \kappa)}(\mathbf{y})$ polynomial. We want to cancel common factors and reorder to remove the $n$ dependence from the bounds on the product, and make it more explicit. Using the Pochhammer symbols, one obtains

$$
\begin{aligned}
\left\langle P_{\lambda}^{1 / \kappa}(\mathbf{y})\right\rangle_{J}= & \prod_{i=1}^{\ell(\lambda)} \frac{(a+1+\kappa(n-i))_{\lambda_{i}}}{(a+b+2+\kappa(2 n-i-1))_{\lambda_{i}}} \\
& \times \prod_{1 \leq i<j \leq \ell(\lambda)} \frac{(\kappa(j-i+1))_{\lambda_{i}-\lambda_{j}}}{(\kappa(j-i))_{\lambda_{i}-\lambda_{j}}} \prod_{1 \leq i \leq \ell(\lambda)<j \leq n} \frac{(\kappa(j-i+1))_{\lambda_{i}}}{(\kappa(j-i))_{\lambda_{i}}}
\end{aligned}
$$

Clearly the last term can be rewritten as

$$
\begin{aligned}
\left\langle P_{\lambda}^{1 / \kappa}(\mathbf{y})\right\rangle_{J}= & \prod_{i=1}^{\ell(\lambda)} \frac{(a+1+\kappa(n-i))_{\lambda_{i}}}{(a+b+2+\kappa(2 n-i-1))_{\lambda_{i}}} \\
& \times \prod_{1 \leq i<j \leq \ell(\lambda)} \frac{(\kappa(j-i+1))_{\lambda_{i}-\lambda_{j}}}{(\kappa(j-i))_{\lambda_{i}-\lambda_{j}}} \prod_{1 \leq i \leq \ell(\lambda)} \frac{(\kappa(n-i+1))_{\lambda_{i}}}{(\kappa(\ell(\lambda)+1-i))_{\lambda_{i}}}
\end{aligned}
$$

Using the identity (211) this simplifies into:

$$
\left\langle P_{\lambda}^{1 / \kappa}(\mathbf{y})\right\rangle_{J}=\frac{\alpha^{k}}{c(\lambda, \alpha, 1)} \prod_{i=1}^{\ell(\lambda)} \frac{(a+1+\kappa(n-i))_{\lambda_{i}}}{(a+b+2+\kappa(2 n-i-1))_{\lambda_{i}}}(\kappa(n-i+1))_{\lambda_{i}}
$$

which using the relation (72) between the different Jack polynomials, gives the formula (76) in the text. 


\section{Appendix 6: Remark on Moment Formula}

It is interesting to note that the formula $(71,70)$ can be rewritten as:

$$
p_{(k)}(y)=k \alpha \lim _{p \rightarrow 0} \sum_{\lambda,|\lambda|=k} \frac{J_{\lambda}^{(\alpha)}\left(1_{p}\right) J_{\lambda}^{(\alpha)}(y)}{<J_{\lambda}^{(\alpha)}, J_{\lambda}^{(\alpha)}>}
$$

using that $J_{\lambda}^{(\alpha)}\left(1_{p}\right)=\prod_{s \in \lambda}\left(p-l_{\lambda}^{\prime}(s)+\alpha a_{\lambda}^{\prime}(s)\right)$ see Theorem 5.4 in [55] and (10.25) in [54]. This may be compared to the Cauchy identity [55]

$$
\sum_{\lambda} q^{|\lambda|} \frac{J_{\lambda}^{(\alpha)}(\mathbf{y}) J_{\lambda}^{(\alpha)}(\mathbf{x})}{<J_{\lambda}, J_{\lambda}>}=\prod_{i, j}\left(1-q x_{i} y_{j}\right)^{-1 / \alpha}
$$

where the sum is over all partitions. Another important identity is based on the so-called binomial formula [30], and is quoted and used in [31]. It reads

$$
\sum_{\lambda} q^{|\lambda|} \prod_{s \in \lambda}\left(a \alpha-l_{\lambda}^{\prime}(s)+\alpha a_{\lambda}^{\prime}(s)\right) \frac{J_{\lambda}^{(\alpha)}(\mathbf{x})}{<J_{\lambda}, J_{\lambda}>}=\prod_{i=1}^{n}\left(1-q x_{i}\right)^{-a}
$$

and agrees with Cauchy formula setting $a=n / \alpha$ and all $y_{i}=1$.

Finally let us note an alternative way to recover the moment formula $(33,34)$, starting from Equation (2.11)-(2.12) in [60] ${ }^{11}$ and performing some manipulations. One first sets $w=0$ which selects positive signatures, i.e. usual partitions $\lambda$. Next, one applies the relation to $n$ variables $u_{i}$ in $[0,1]$ rather than on the unit circle, and one applies the identities for $n<\ell(\lambda)$ ( $\ell(\lambda)$ is called $N$ there), i.e. we set the remaining variables to zero. Next, one transforms $u_{i} \rightarrow q u_{i}$ and use homogeneity of Macdonald polynomials. One further expands at small $z$ and consider the $O(z)$ term, which is then averaged over the Jacobi measure using the Kadell integral. As we have checked explicitly, this leads, after some algebra, to $(33,34)$.

\section{Appendix 7: Distribution of the Value of the Minimum}

It is useful to recall the analysis of [12] for the PDF of the value of the maximum, but in a much more concise form, and further elaborate to the present cases. We will not attempt at rigor and refer the reader to [40-43] for steps in that direction.

\section{Main Result}

Let us first present the quick and dirty version, directly at $\beta=1$ and give later a better justification starting from $\beta<1$ and using duality. The positive integer moments of the reduced partition sum $z_{\beta}=\Gamma\left(1-\beta^{2}\right) Z_{\beta}$ of the model (47) on the interval are given by the Selberg integral (29)

$$
\overline{z_{\beta}^{n}}=\left.S l_{n}(\kappa, a, b)\right|_{\kappa=-\beta^{2}}
$$

which is Eq. (7) in [12]. Let us use the identity

$$
\prod_{j=1}^{n} \Gamma(z-j)=\frac{G(z)}{G(z-n)}
$$

11 we thank A. Borodin for pointing out this reference and useful comments about this formula. 
in terms of the Barnes function $G(x)$, valid for positive integer $n$ and complex $z$. Brutally replace in the Selberg integral setting $\beta=1$. For definiteness the argument in the last $\Gamma$-function in (29) may be slighlty shifted before the replacement, and taken back to zero afterwards. This leads to

$$
\overline{z_{1}^{n}}=\overline{e^{-n f_{1}}}=S(n)=\frac{G(1) G(2+a) G(2+b) G(4+a+b-2 n)}{G(1-n) G(2+a-n) G(2+b-n) G(4+a+b-n)}
$$

which is now continued to complex $n$, with $\operatorname{Re}(n)<1$. Here $a, b$ denote the value of the (possibly temperature-dependent) parameters at $\beta=1$. Note that $a, b$ may also depend on $n$, e.g. see below the fBm example.

Freezing (see below) states that the PDF of the random variable

$$
y_{\beta}=f_{\beta}-G / \beta
$$

where $G$ is a unit Gumbel random variable, independent of $f_{\beta}$, is independent of $\beta$ for all $\beta \geq 1$. This implies ${ }^{12}$

$$
V_{m}=\text { in law } f_{1}-G
$$

hence the PDF of $V_{m}$, can be obtained by inverse Laplace transform of (236) convoluted with Gumbel, i.e.

$$
\begin{aligned}
& \overline{e^{-n V_{m}}}=\Gamma(1-n) S(n) \\
& Q\left(V_{m}\right)=L T_{n \rightarrow V_{m}}^{-1} \Gamma(1-n) S(n)
\end{aligned}
$$

Using that:

$$
\partial_{z} \ln G(z)=\frac{1}{2}(1+\ln (2 \pi))-z+(z-1) \psi(z)
$$

One easily obtain the cumulants of $V_{m}$. One must distinguish two cases according to whether the Jacobi variables $a, b$ (at $\beta=1$ ) depend on $n$ or not (and similarly when performing the inverse Laplace transform).

In the case where $a, b$ are $n$-independent (LCGP with edge charges, and GUE-CP) one finds, for $p \geq 1$

$$
{\overline{V_{m}^{p}}}^{c}=\left(2^{p}-1\right) \phi_{p}(4+a+b)-\phi_{p}(2+a)-\phi_{p}(2+b)+\gamma_{p}
$$

where

$$
\begin{aligned}
& \phi_{p}(z)=(p-1) \psi_{p-2}(z)+(z-1) \psi_{p-1}(z) \\
& \gamma_{p}=(-1)^{p}(p-1) !(\zeta(p)+\zeta(p-1)), \quad p \geq 3 \\
& \gamma_{2}=\gamma_{E}+\frac{\pi^{2}}{6}, \quad \gamma_{1}=-\gamma_{E}-\ln 2 \pi
\end{aligned}
$$

where we used, for $p \geq 2, \psi_{p}(1)=(-1)^{p+1} \zeta(p+1) p$ !, and the cumulants of the Gumbel distribution, $<G^{p}>^{c}=(p-1) ! \zeta(p)$. The results for the LCGP with edge charges are then obtained by setting $a=\bar{a}, b=\bar{b}$, and for the GUE-CP $a=b=1$. In this case the domain of parameters where (227) yields a bona-fide, i.e. positive and well defined PDF, has been discussed in [12]. For the GUE-CP one finds

$$
{\overline{V_{m}^{2}}}^{c}=-\frac{629}{48}+2 \pi^{2}=6.63504
$$

\footnotetext{
12 We thank X. Cao for a stimulating discussion on this point. Note that this is equivalent to the relation between cumulants given in (24) of [12].
} 


$$
\begin{aligned}
& {\overline{V_{m}^{3}}}^{c}=-64 \zeta(3)+\frac{50549}{864}+\frac{4 \pi^{2}}{3}=-5.26638 \\
& {\overline{V_{m}^{4}}}^{c}=-72 \zeta(3)-\frac{423301}{1152}+\frac{24 \pi^{4}}{5}=13.5668
\end{aligned}
$$

in agreement with the study [21]. ${ }^{13}$

For the second case let us discuss the $\mathrm{fBm} 0$, where $a=2 \beta^{2} n \rightarrow 2 n$ should be inserted. This problem is much more delicate. Indeed for $a$ sufficiently negative we know that a binding transition occurs at the boundary [12]. The naive application of the method would yield, for the Laplace transform of the PDF of the value of the minimum $V_{m}$

$$
\overline{e^{-n V_{m}}}=\Gamma[1-n] \frac{G(1) G(2+2 n) G(2) G(4)}{G(1-n) G(2+n) G(2-n) G(4+n)}
$$

where $G(1)=G(2)=1$ and $G(4)=2$. This however cannot be the LT of a positive probability, since it vanishes at $n=-1$ and convexity is violated around $n<-.22 \ldots$. Worse, we find that the Taylor coefficient of $n^{4}$ is -0.116681 , thus cumulants cannot be obtained. The origin of this problem is clear. Contrarily to the other cases the value of the potential is fixed at $x=0, V(0)=0$. Hence $V_{m}$ cannot be positive, it is either $V_{m}<0$ or pinned at $V_{m}=0$ in which case $x_{m}=0$. When $n$ is negative, the moment (236) gives a lot of weight to higher (less negative values) of $V_{m}$, and eventually it reaches $V_{m}=0$ corresponding to the above mentioned "binding transition". This explains why the analytical structure of (236) is badly behaved on the negative $n$ side, and cannot be the proper analytical continuation in that region. At the minimum one needs to better take into account a possible delta-function weight at $V_{m}=0$. Fixing this problem, and finding the correct analytical continuation for this case, seems challenging and is left for future studies. ${ }^{14}$

\section{Duality and Freezing}

Let us now consider $\beta<1$ where analytical continuation can be studied with more care. There exists a function $\tilde{G}_{\beta}(x)\left(\right.$ see $\left.^{15}\right)$ such that (222) generalizes to

$$
\prod_{j=1}^{n} \Gamma\left(\beta z-j \beta^{2}\right)=\frac{\tilde{G}_{\beta}(z)}{\tilde{G}_{\beta}(z-n \beta)}
$$

where $\tilde{G}_{\beta}(z)=A_{z, \beta} G_{\beta}(z)$ and $G_{\beta}(z)$ is the function introduced in [12] which satisfies the duality invariance $G_{\beta}(z)=G_{1 / \beta}(z)$, and $G_{1}(z)=G(z), A_{z, 1}=1$. The precise value of $A_{z, \beta}=\beta^{\frac{z^{2}}{2}-\frac{z}{2}\left(\beta+\frac{1}{\beta}\right)}(2 \pi)^{z\left(\frac{1}{2 \beta}-\frac{1}{2}\right)}$. Using this formula with $a=\beta \bar{a}$ and $b=\beta \bar{b}$ we obtain

\footnotetext{
13 The analogous formula for the Laplace transform there contains an additional global prefactor $\pi^{-2 n} 2^{8 n-2 n^{2}}$, which we have neglected here-as it is immaterial for the moments of the position of the maximum - but needs to be restored for comparison with the value of the maximum in the GUE-CP problem). It shifts the value of the second cumulant by $-4 \ln 2$ as compared to (233), but leaves the higher cumulants unchanged. We thank Nick Simm for help in clarifying this point.

14 Note also the regularization dependent factor $\eta^{\beta^{2} n^{2}}$ in the formula (53) for the moments, omitted here, but which, when reintroduced, helps cure the convexity problem mentioned here. Whether this allows a full solution of the problem (in particular whether the cumulants of order larger than 2 are correctly predicted by (236), is presently unclear and left for future study. We thank D. Ostrovsky for a discussion about these issues.

15 Note that the function $\tilde{G}_{\beta}(x)$ is related to the Alexeiewsky-Barnes $\mathrm{G}$ function noted $G(z \mid \tau)$ defined in Eq. (8) of [41] (and studied there) as follows $\tilde{G}_{\beta}(\beta x) / \tilde{G}_{\beta}(\beta)=\left.G(x \mid \tau)\right|_{\tau=1 / \beta^{2}}$.
} 
from the Selberg integral (29)

$$
\begin{aligned}
& \overline{z_{\beta}^{n}}=\overline{e^{-n \beta f_{\beta}}}=S_{\beta}(n) \\
& =\frac{\tilde{G}_{\beta}\left(\frac{1}{\beta}\right) \tilde{G}_{\beta}\left(\frac{1}{\beta}+\beta+\bar{a}\right) \tilde{G}_{\beta}\left(\frac{1}{\beta}+\beta+\bar{b}\right) \tilde{G}_{\beta}\left(\frac{2}{\beta}+2 \beta+\bar{a}+\bar{b}-2 \beta n\right)}{\tilde{G}_{\beta}\left(\frac{1}{\beta}-\beta n\right) \tilde{G}_{\beta}\left(\frac{1}{\beta}+\beta+\bar{a}-\beta n\right) \tilde{G}_{\beta}\left(\frac{1}{\beta}+\beta+\bar{b}-\beta n\right) \tilde{G}_{\beta}\left(\frac{2}{\beta}+2 \beta+\bar{a}+\bar{b}-\beta n\right)}
\end{aligned}
$$

which is now legitimate for $\beta$ small enough at fixed $n$. Now, using that $\tilde{G}_{\beta}(z)=\tilde{G}_{\beta}(z+$ $\beta) / \Gamma(\beta z)$, and upon a trivial shift $z=\tilde{z}(2 \pi)^{1-\beta}$ (and further ignoring the tilde) we note that it can be rewritten as

$$
\overline{z_{\beta}^{n}}=\overline{e^{-n \beta f_{\beta}}}=\Gamma\left(1-\beta^{2} n\right) \bar{S}_{\beta}(\beta n)
$$

where

$$
\begin{aligned}
& \bar{S}_{\beta}(\beta n) \\
& =\frac{G_{\beta}\left(\beta+\frac{1}{\beta}\right) G_{\beta}\left(\frac{1}{\beta}+\beta+\bar{a}\right) G_{\beta}\left(\frac{1}{\beta}+\beta+\bar{b}\right) G_{\beta}\left(\frac{2}{\beta}+2 \beta+\bar{a}+\bar{b}-2 \beta n\right)}{G_{\beta}\left(\beta+\frac{1}{\beta}-\beta n\right) G_{\beta}\left(\frac{1}{\beta}+\beta+\bar{a}-\beta n\right) G_{\beta}\left(\frac{1}{\beta}+\beta+\bar{b}-\beta n\right) G_{\beta}\left(\frac{2}{\beta}+2 \beta+\bar{a}+\bar{b}-\beta n\right)}
\end{aligned}
$$

is a fully duality invariant function $S_{\beta}(x)=S_{1 / \beta}(x)$. Here we are using the same definition of duality invariance as in (90), i.e. the combination $\beta n$ is duality invariant (since $n^{\prime}=$ $\left.n \beta^{2}=n \beta / \beta^{\prime}\right)$. We note that the factor $\Gamma\left(1-\beta^{2} n\right)$ corresponds exactly to the moments of the (simpler) $\log$-circular ensemble [11], in other words in that case the factor $\bar{S}(\beta n)=1$.

So we see from (239), and since $n \beta$ is duality invariant, that the free energy random variable is not duality invariant. However it is now trivial to see what one must do to make (239) fully duality invariant, namely multiply it by $\Gamma(1-n)$, which is the image of $\Gamma\left(1-n \beta^{2}\right)$ under duality. And this amounts precisely to a convolution by an independent Gumbel variable. Hence defining the random variable

$$
y_{\beta}=f_{\beta}-G / \beta
$$

we have that

$$
\overline{e^{-n \beta y_{\beta}}}=\Gamma(1-n) \overline{e^{-n \beta f_{\beta}}}=\Gamma(1-n) \Gamma\left(1-n \beta^{2}\right) \bar{S}(\beta n)
$$

and the random variable $y_{\beta}$ is fully duality invariant (meaning all its exponential moments are). Now the freezing duality conjecture states that the PDF of the variable $y_{\beta}$ freezes at $\beta=1$, leading to the results for the PDF of the minimum $V_{m}$, displayed in the previous subsection (since $G_{1}(z)=\tilde{G}_{1}(z)=G(z)$ all formula trivially match). Note that the CDF of $y_{\beta}$ is $1-g_{\beta}(y)$ where $g_{\beta}(y)=\overline{e^{-e^{\beta\left(y-f_{\beta}\right)}}}$, hence duality invariance and freezing of (240) is equivalent to stating that the full function $g_{\beta}(y)$ is duality invariant and freezes.

Finally note that all which is needed for duality invariance and freezing in this class of models, is that, as discussed in (95), $\bar{a}$ and $\bar{b}$ are duality invariant functions of $\beta$ and (when it happens) of $\beta n$, which is the case for all three examples studied here.

\section{References}

1. Fyodorov, Y.V., Khoruzhenko, B.A., Simm, N.J.: Fractional Brownian Motion with Hurst Index $H=0$ and the Gaussian Unitary Ensemble (2013). e-preprint arXiv:1312.0212 
2. Fyodorov, Y.V., Keating, J.P.: Freezing transitions and extreme values: random matrix theory, $\zeta(1 / 2+i t)$ and disordered landscapes. Philos. Trans. R. Soc. Lond. Ser. A 372(2007), 20120503 (2014)

3. Duplantier, B., Rhodes, R., Sheffield, S., Vargas, V.: Log-Correlated Gaussian Fields: An Overview (2014). epreprint arXiv: 1407.5605

4. Rhodes, R., Vargas, V.: Gaussian Multiplicative Chaos and applications: an overview. Probab. Surv., 11, 315-392 (2014). doi:10.1214/13-PS218

5. Kistler, N.: Derrida's random energy models. From spin glasses to the extremes of correlated random fields (2014). e-preprint arXiv: 1412.0958

6. Carpentier, D., Le Doussal, P.: Glass transition of a particle in a random potential, front selection in nonlinear renormalization group, and entropic phenomena in Liouville ane sinh-Gordon models. Phys. Rev. E 63, 026110 (2001)

7. Sheffield, S.: Gaussian free fields for mathematicians. Prob. Theor. Relat. Fields 139(3-4), 521-541 (2007)

8. Antal, T., Droz, M., Györgyi, G., Racz, Z.: 1/f noise and extreme value statistics. Phys. Rev. Lett. 87, $240601(2001)$

9. Fyodorov, Y.V., Le Doussal, P., Rosso, A.: Counting function fluctuations and extreme value threshold in multifractal patterns: the case study of an ideal 1/f noise. J. Stat. Phys. 149, 898-920 (2012)

10. Astala, K., Jones, P., Kupiainen, A., Saksman, E.: Random conformal weldings. Acta Math. 207, 203-254 (2011)

11. Fyodorov, Y.V., Bouchaud, J.P.: Freezing and extreme-value statistics in a random energy model with logarithmically correlated potential. J. Phys. A 41(37), 372001 (2008)

12. Fyodorov, Y.V., Le Doussal, P., Rosso, A.: Statistical mechanics of logarithmic REM: duality, freezing and extreme value statistics of 1/f noises generated by Gaussian free fields. J. Stat. Mech. Theor. Exp. 10, P10005 (2009)

13. Fyodorov, Y.V., Hiary, G.H., Keating, J.P.: Freezing transition, characteristic polynomials of random matrices, and the Riemann zeta-function. Phys. Rev. Lett. 108, 170601 (2012)

14. Cao, X., Rosso, A., Santachiara, R.: Extreme value statistics of $2 \mathrm{~d}$ Gaussian Free Field: effect of finite domains. J. Phys. A 49, 02LT02 (2016)

15. Fyodorov, Y.V., Giraud, O.: High values of disorder-generated multifractals and logarithmically correlated processes. Chaos Soliton Fract. 74: 15-26 (2015). e-preprint arXiv:1407.4035

16. Bramson, M., Zeitouni, O.: Tightness of the recentered maximum of the two-dimensional discrete Gaussian free field Comm. Pure Appl. Math. 65, 1-20 (2012)

17. Bramson, M., Ding, J., Zeitouni, O.: Convergence in law of the maximum of the two-dimensional discrete Gaussian free field. Commun. Pure Appl. Math 69, 62-123 (2016)

18. Bourgade, P., Kuan, J.: Strong Szego asymptotics and zeros of L-functions. Commun. Pure Appl. Math 67, 1028-1044 (2014)

19. Ding, J., Zeitouni, O.: Extreme values for two-dimensional discrete Gaussian free field. Ann. Probab. 42(4), 1480-1515 (2014)

20. Ding, J., Roy, R., Zeitouni, O.: Convergence of the centered maximum of log-correlated Gaussian fields. e-preprint arXiv:1503.04588 (2015)

21. Fyodorov, Y.V., Simm, N.J.: On the distribution of maximum value of the characteristic polynomial of GUE random matrices (2015). e-preprint arXiv:1503.07110

22. Arguin, L.-P., Zindy, O.: Poisson-Dirichlet statistics for the extremes of a log-correlated Gaussian field. Ann. Appl. Probab. 24, 1446-1481 (2014)

23. Madaule, T., Rhodes, R., Vargas, V.: Glassy phase and freezing of log-correlated Gaussian potentials. Ann. Appl. Probab. 26(2), 643-690 (2016)

24. Arguin, L.-P., Zindy, O.: Poisson-Dirichlet statistics for the extremes of the two-dimensional discrete Gaussian free field. EJP 20, 3077 (2015)

25. Arguin, L.-P., Ouimet, F.: Extremes of the two-dimensional Gaussian free field with scale-dependent variance. e-preprint arXiv:1508.06253

26. Arguin, L.P., Belius, D., Harper, A.J.: Maxima of a randomized Riemann Zeta function, and branching random walks. e-preprint arXiv:1506.00629

27. Webb, C.: The Characteristic Polynomial of a Random Unitary Matrix and Gaussian Multiplicative Chaos-the L2-Phase. e-preprint arXiv:1410.0939 (2014)

28. Webb. C,: Global spectral fluctuations in the Gaussian Unitary Ensemble. e-preprint arXiv: 1510.05867

29. Dumitriu, I., Edelman, A.: Matrix models for beta ensembles. J. Math. Phys. 43, 5830-5847 (2002)

30. Forrester, P.J.: Log-Gases and Random Matrices. London Mathematical Society Monographs. Princeton University Press, Princeton (2010)

31. Mezzadri, F., Reynolds, A.K.: Moments of the eigenvalue densities and of the secular coefficients of $\beta$-ensembles. e-preprint arXiv: 1510.02390 
32. Anderson, G.W., Guionnet, A.: An Introduction to Random Matrices. Cambridge University Press, Cambridge (2009)

33. Mehta, M.L.: Random Matrices, 3rd edn. Academic Press, San Diego (2004)

34. Pastur, L., Shcherbina, M.: Eigenvalue Distribution of Large Random Matrices. AMS, Providence (2011)

35. Krasovsky, I.V.: Correlations of the characteristic polynomials in the Gaussian unitary ensemble or a singular Hankel determinant. Duke Math. J. 139(3), 581-619 (2007)

36. Forrester, P.J., Frankel, N.E.: Applications and generalizations of Fisher-Hartwig asymptotics. J. Math. Phys. 45, 2003-2028 (2004)

37. Garoni, T.M.: On the asymptotics of some large Hankel determinants generated by Fisher-Hartwig symbols defined on the real line. J. Math. Phys. 46, 043516 (2005)

38. Mandelbrot, B.B., van Ness, J.W.: Fractional Brownian motions. Fractional noises and applications. SIAM Rev. 10(4), 422-437 (1968)

39. Forrester, P.J., Warnaar, S.O.: The importance of the selberg integral. Bull. Am. Math. Soc. 45, 489-534 (2008)

40. Ostrovsky, D.: Mellin transform of the limit lognormal distribution. Commun. Math. Phys. 288, 287-310 (2009)

41. Ostrovsky, D.: Selberg integral as a meromorphic function. Int. Math. Res. Not., 2012 (2012)

42. Ostrovsky, D.: Theory of Barnes beta distributions. Electron. Commun. Prob. 18(59), 116 (2012)

43. Ostrovsky, D.: On Barnes beta distributions, Selberg Integral and Riemann Xi. Forum Math. (2014). doi:10.1515/forum-2013-0149

44. Savin, D.V., Sommers, H.-J.: Shot noise in chaotic cavities with an arbitrary number of open channels. Phys. Rev. B. 73, 081307(R) (2006)

45. Savin, D.V., Sommers, H.-J., Wieczorek, W.: Nonlinear statistics of quantum transport in chaotic cavities. Phys. Rev. B 77, 125332 (2008)

46. Khoruzhenko, B.A., Savin, D.V., Sommers, H.-J.: Systematic approach to statistics of conductance and shot-noise in chaotic cavities. Phys. Rev. B 80, 125301 (2009)

47. Vivo, P., Vivo, E.: Transmission eigenvalue densities and moments in chaotic cavities from random matrix theory. J. Phys. A 41, 122004 (2008). Fast Track Communication

48. Mezzadri, F., Simm, N.J.: Tau-function theory of quantum chaotic transport with beta=1,2,4. Commun. Math. Phys. 324, 465-513 (2013)

49. Fyodorov, Y.V., Le Doussal, P., Rosso, A.: Freezing transition in decaying Burgers turbulence and random matrix dualities. Europhys. Lett. 90, 60004 (2010)

50. Subag, E., Zeitouni, O.: Freezing and decorated Poisson point processes. Commun. Math. Phys. 337(1), 55-92 (2015)

51. Zamolodchikov, A., Zamolodchikov, A.: Conformal bootstrap in Liouville field theory. Nucl. Phys. B 477, 577-605 (1996)

52. Kadell, K.W.J.: The Selberg-Jack symmetric functions. Adv. Math. 130, 33-102 (1997)

53. Kuznetsov, V.B., Sahi, S.: Jack, Hall-Littlewood and Macdonald Polynomials. Contemporary Mathematics, vol. 417. American Mathematical Society, Providence (2006)

54. Macdonald, I.G.: Symmetric Functions and Hall Polynomials. Oxford Mathematical Monographs, 2nd edn. Oxford University Press, New York (1995)

55. Stanley, R.P.: Some combinatorial properties of Jack symmetric functions. Adv. Math. 77, 76-115 (1989)

56. Vassilieva, E.A.: Jack's connection coefficients - First results and a generalization of a formula by Denes. J. Algebr. Comb. 42(1), 51-71 (2015)

57. Borodin, A., Olshanski, G.: Infinite random matrices and ergodic measures. Comm. Math. Phys. 223(1), 87-123 (2001)

58. Negut, A: Operators on symmetric polynomials e-preprint. arXiv:1310.3515

59. Borodin, A., Gorin, V.: General beta Jacobi corners process and the Gaussian Free Field. Commun. Pure Appl. Math. (to appear). arXiv:1305.3627

60. Olshanskii, G.I.: Probability measures on dual objects to compact symmetric spaces and hypergeometric identities. Funct. Anal. Appl. 37(4), 281-301 (2003) 